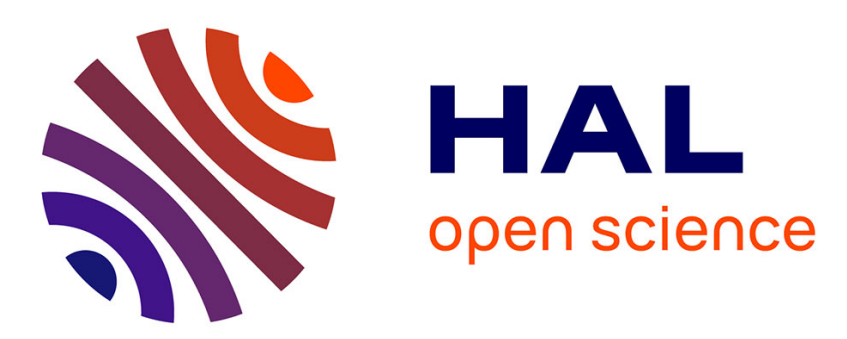

\title{
Quasi-oralité de l'ecriture électronique et sentiment de communauté dans les débats scientifiques en ligne
}

\author{
Philippe Hert
}

\section{To cite this version:}

Philippe Hert. Quasi-oralité de l'ecriture électronique et sentiment de communauté dans les débats scientifiques en ligne. Réseaux: communication, technologie, société, 1999, 17 (97). sic_00000517

\section{HAL Id: sic_00000517 \\ https://archivesic.ccsd.cnrs.fr/sic_00000517}

Submitted on 15 Jul 2003

HAL is a multi-disciplinary open access archive for the deposit and dissemination of scientific research documents, whether they are published or not. The documents may come from teaching and research institutions in France or abroad, or from public or private research centers.
L'archive ouverte pluridisciplinaire HAL, est destinée au dépôt et à la diffusion de documents scientifiques de niveau recherche, publiés ou non, émanant des établissements d'enseignement et de recherche français ou étrangers, des laboratoires publics ou privés. 


\section{QUASI-ORALITE DE L'ECRITURE ELECTRONIQUE ET SENTIMENT DE COMMUNAUTE DANS LES DEBATS SCIENTIFIQUES EN LIGNE}

Philippe HERT 

$\mathrm{C}$

et article propose d'analyser le processus de construction d'un sentiment de communauté à travers les interactions écrites se produisant au sein de forums de discussions sur Internet. Pour expliquer cette dynamique, j'adopterai une perspective à la fois sociologique et sémiotique. J'introduirai la notion de quasi-oralité de l'écriture à travers une réflexion sur l'utilisation de l'écriture électronique par un collectif.

Les usages des listes et forums de discussions électroniques font l'objet d'une connaissance plus ou moins intuitive pour quiconque ayant pratiqué ce type d'échange. Pour les autres, il s'agit souvent de pratiques ésotériques, d'activités de "cybernautes" vivant apparemment dans un univers différent du leur. Entre ces deux positions, nous manquons largement de réflexions permettant de faire la médiation entre des pratiques minoritaires, non réellement analysées, et la majorité qui n'a souvent que les discours de la nouveauté de ces modes de communication sur lesquels s'appuyer. Banalisation d'une part, nouveauté radicale de l'autre. Or, si l'on peut parler de banalisation, c'est effectivement parce que les nouvelles pratiques s'inscrivent dans un tissu social déjà structuré. D'autre part, si l'on parle également de nouveauté, c'est parce qu'il y a bien des "effets" de ces technologies sur la communication. Penser ces effets n'est pas aisé, car le risque de tomber dans une forme de déterminisme technologique n'est pas absent: la technologie induit des effets sur la communication et semble donc déterminer les relations sociales à travers ce support. Ainsi, Kiesler et

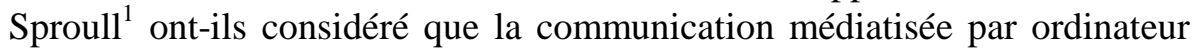
était déterminée par les propriétés de la machine. Parce que les personnes qui utilisent ce média ne peuvent se voir, s'entendre et percevoir leurs attitudes, elles peuvent difficilement utiliser des éléments du contexte d'énonciation. Selon ces auteurs, ce manque d'indices sociaux aboutit à un plus grand anonymat et permet une participation plus large que dans des interactions menées en face à face. Parallèlement à cela, le temps mis pour atteindre un consensus est augmenté. Selon cette perspective, le média détermine donc le type d'interaction qui se produit.

\footnotetext{
${ }^{1}$. KIESLER et SPROULL, 1991.
} 
Camper sur une telle position serait cependant trop simpliste : les effets dépendent énormément du rapport qu'établissent les individus avec le dispositif. Ce rapport implique aussi bien les aspects stratégiques de la communication (tous les aspects rhétoriques de la communication) que technologiques (ce que Michel de Fornel appelle les "affordances" d'une technologie ${ }^{2}$ ), cognitives (leur familiarité avec le dispositif, les compétences acquises), sémiotiques (le développement de systèmes symboliques particuliers à ce mode de communication, notamment à travers le rapport entre l'écriture et l'oralité) ou encore sociales (la position dans le cadre d'un débat, la défense de certains intérêts, le contexte de l'échange et la présence de certains acteurs). C'est l'ensemble de ces dimensions qui produit un effet à travers une hybridation des aspects sociaux et technologiques (on peut d'ailleurs inclure les procédés rhétoriques parmi les technologies employées dans le rapport développé par les usagers avec le dispositif technique ${ }^{3}$.

L'analyse présentée ici se fonde sur deux ethnographies d'interactions entre scientifiques à travers Internet. Il s'agit essentiellement d'un débat qui s'est déroulé dans une liste de discussion_électronique dédiée à la communauté STS (Science-Technologie-Société) ${ }^{4}$. Le second terrain d'étude sera présenté comme un contrepoint et concerne un ensemble d'échanges électroniques locaux entre chimistes d'une université française. De semblables analyses ethnographiques d'interactions électroniques ont déjà été menées mais leur contenu n'avait pas de lien avec l'activité scientifique $^{\mathbf{5}}$. Une recherche du CRICT ${ }^{6}$ analyse par exemple les débats entre scientifiques en biologie concernant l'étude du génome humain. Cependant, ces travaux ne rendent pas compte de la manière dont la matérialité du support de communication interagit avec ce que ses utilisateurs y construisent à travers une écriture qu'ils inventent. Pour cela, il est peut-être nécessaire de trouver de nouveaux concepts propres à nous permettre de penser les pratiques de communication qui se développent à travers cet outil. C'est pourquoi j'introduirai ici la notion de quasi-oralité,

2. FORNEL (de), 1994.

${ }^{3}$. Sur l'hybridation des pratiques sociales et des innovations technologiques, voir par exemple BIJKER et LAW, 1992.

${ }^{4}$. STS : Science Technologie et Société est le nom sous lequel diverses recherches sur la construction sociale des sciences et des techniques se retrouvent.

5. BAYM, 1995 ; AYCOCK et BUCHIGNANI, 1995 ; VIDAL, 1999, par exemple.

${ }^{6}$. Center for Research into Innovation, Culture and Technology, Brunel, University of WestLondon. Voir HINE, 1996. 
sachant qu'elle demande des développements beaucoup plus approfondis qu'il n'est possible de mener ici pour être définie avec précision (je renverrai simplement, dans la mesure du possible, à d'autres travaux).

Le choix d'analyser les pratiques de communication de scientifiques et non pas des échanges portant sur des sujets d'actualité ou des thèmes de société par exemple, est lié au type de participants et aux contenus abordés. L'usage d'Internet par les scientifiques se prête à une analyse approfondie, contrairement à d'autres groupes sociaux, car il s'agit de communautés

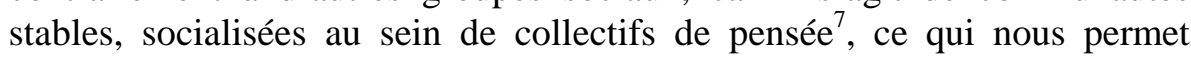
d'établir un certain nombre d'hypothèses sur le contexte de réception. Il est également possible de constituer ainsi un corpus précis et convenablement délimité. De plus, une étude ethnographique des interactions électroniques ne saurait faire l'économie d'une prise en considération de tous les aspects de la communauté étudiée. Il convient donc de pouvoir également rencontrer les personnes dans leur contexte de travail. Cette approche, qui ne se dispense pas "d'aller voir sur le terrain", permet de ne pas s'en tenir simplement aux discours.

Les forums de discussions utilisés par les scientifiques sont la plupart du temps publics et librement accessibles à travers Internet. Ce mode de communication entre scientifiques semble constituer un moyen idéal pour créer des lieux de débats. Il remplace en effet avantageusement les forums d'expression qui existent dans certaines revues de recherche (tribunes, courrier des lecteurs, droits de réponses...), dont l'utilisation reste modérée en raison des délais très longs de publication. Cependant, la portée de ces espaces de discussions dans le processus de transformation de l'information scientifique en savoir scientifique n'est pas claire. Bruce Lewenstein 8 nous précise les limites des apports de ces forums pour la connaissance en science. L'auteur a comparé ces modes électroniques de diffusion de l'information avec les moyens traditionnels, au moment de la controverse sur la fusion froide. Cette controverse est un exemple d'utilisation de ces nouveaux modes de diffusion de l'information scientifique dans un contexte de crise. L'étude de Lewenstein a montré que ces forums de débats et d'échanges étaient des outils inefficaces pour produire un savoir scientifique, bien qu'ils aient permis aux scientifiques à travers le monde d'obtenir des informations et des données plus précises sur l'expérience de

7. Au sens de Fleck : voir FLECK, 1979.

${ }^{8}$. LEWENSTEIN, 1995. 
Fleishmann et Pons. Les raisons de cet échec tenaient principalement à la grande quantité d'informations non pertinentes pour les scientifiques contenues dans les messages concernant la controverse, ainsi que l'importante difficulté rencontrée pour nuancer, évaluer ou recontextualiser les informations qui circulaient.

Ce premier élément d'analyse indique bien une difficulté dans la construction d'un sens commun à travers les interactions électroniques. Nous interrogerons cette difficulté à partir d'une réflexion sur l'écriture électronique. Cette écriture peut être qualifiée de quasi orale, et renvoie à la construction sociale du sens du collectif propre à un groupe. Le fait que ce groupe soit virtuel dans le forum de discussion, mais ait également une existence sociale réelle (la communauté STS dans notre cas), pose de nouvelles questions sur le lien existant entre un collectif humain et les ressources qu'il peut mobiliser pour construire une compréhension collective sur lui-même et sur sa manière d'interpréter le monde. C'est la parole qui circule au sein d'un collectif qui permet de construire ce sens commun. Que se passe-t-il lorsque cette parole fait défaut? Telle est la question à laquelle nous tenterons de répondre à travers l'analyse de la seule ressource disponible dans un débat électronique : l'écriture électronique.

L'idée de quasi-oralité qui sera développée ici permettra d'analyser la nature du sentiment de collectivité qui résulte de la construction sociale de débats électroniques. L'oralité dans l'écriture électronique dont il sera question ne renvoie pas simplement au style interactif des messages qui est parfois très proche d'échanges oraux (ton informel, dépendance du contexte, phrases inconsistantes et manque d'attention accordée à l'orthographe, réactions immédiates à des messages envoyés, etc.). Ce n'est pas là la propriété essentielle de l'écriture quasi orale telle que je la définirai ici; il s'agira davantage d'une utilisation de l'écriture qui ne mime pas forcément l'échange oral, mais qui tente d'étendre l'écriture aux fonctions de l'oralité, et que nous tenterons de voir en détail. Autrement dit, il s'agit ici d'examiner les tentatives mises en œuvre par les participants à des débats électroniques pour conférer à l'écriture une capacité à créer un sens du collectif.

Précisons également tout de suite quel peut être l'enjeu particulier de l'usage de tels espaces de dialogues pour une communauté scientifique. Les conférences électroniques permettent à tous les membres de la communauté de s'exprimer au niveau par exemple d'un débat de nature épistémologique, 
comme nous le verrons avec le second cas étudié ici, et, surtout, de faire ressortir les enjeux conjoncturels d'une communauté de recherche qui n'apparaissent pas dans les articles publiés. Dans les débats électroniques, ce qui peut se manifester, comme d'ailleurs lors de colloques ou de rencontres informelles, c'est ce discours politique, ou le métadiscours conjoncturel, portant sur la définition des questions prioritaires de recherche qui peuvent apporter une avancée dans une communauté de recherche définie. Mais encore s'agit-il de préciser les conditions, liées au dispositif autant qu'à la communauté des utilisateurs, pour qu'une socialisation de ce qui se formule puisse se produire. Si un tel espace d'interlocution existe en fait en permanence au sein d'un laboratoire ${ }^{9}$ il peut également se constituer entre équipes, entre laboratoires. Les outils de communication utilisant Internet peuvent-ils alors jouer le rôle d'un tel espace d'interlocution et fonctionner comme point de départ ou de relais pour des collaborations, sachant qu'un tel espace est avant tout un espace de socialisation communautaire et un espace de parole $\square^{10}$ ? L'analyse des deux débats présentés dans cet article montrera l'échee d'une telle démarche dans le premier cas et un relatif succès dans le second. La notion d'écriture quasiorale nous permettra de préciser les limites de ces espaces d'interaction, à la fois pour construire un savoir commun et pour développer un sens de la communauté.

\section{LA QUASI-ORALITÉ DE L'ÉCRITURE ÉLECTRONIQUE}

\section{Parole et lien social}

Comment l'écriture s'accomode-t-elle du travail de construction de sens commun à un collectif lorsque l'échange oral, direct, fait défaut? En effet, comment parler d'une expérience commune, de pratiques de socialisation fédératrices, d'une construction collective de sens lorsque le seul moyen d'échange n'est pas la parole mais l'écriture ? Pour éclaircir cette question, un détour par les fonctions respectives de l'oral et de l'écrit est nécessaire.

Dans notre civilisation, l'écrit sert de référence, et l'oralité est toujours comprise par rapport à l'écrit. Il s'agit là moins d'une opposition entre oral et écrit que d'un enrichissement de l'oralité par une compréhension de la

\footnotetext{
9 . Voir par exemple LYNCH, 1985.

${ }^{10}$. TRAWEEK, 1988.
} 
manière par laquelle les effets de l'oral sont créés (en particulier tout l'art de la rhétorique). Cette compréhension de la dynamique orale s'effectue à partir d'une manière écrite de penser et de voir les mots : étudier l'utilisation des mots en situation et leur organisation suppose un recul qui n'existe pas lorsqu'on parle ou lorsqu'on écoute un discours sans l'aide de notes écrites, comme nous l'a très bien montré Goody $\square$

Alors que l'écrit joue un rôle central dans notre civilisation, Walter Ong remarque que les nouveaux médias (télévision, radio, téléphone) font réémerger une oralité dans la communication. Mais cette oralité est très différente de celle qui existe pour les groupes humains de culture orale (c'est-à-dire sans connaissance de l'écriture) et que Ong appelle oralité primaire. En effet, cette nouvelle oralité, qualifiée de secondaire, est dépendante de l'écriture. Par conséquent, elle ne joue pas du tout le même rôle que l'oralité définie comme un mode central de communication dans un groupe humain ${ }^{13}$.

L'écriture trahit le groupe par la dimension privée de sa production : auteur et lecteur sont absents l'un à l'autre, situés dans des contextes différents, ne participant pas d'une énonciation conjointe. Mais surtout, l'écriture met en place un rapport d'autorité entre auteur et lecteur. Dans ces conditions, il n'est possible que de mimer dans l'écriture l'échange qui engage la parole de chacun, de reconstruire les propriétés de l'échange oral dans ce qui se sait écriture mais qui tente de redéfinir le rapport auteur/lecteur en se référant aux formes du dialogue oral ${ }^{44}$. Dès lors, on voit apparaître le rapport pouvant exister entre les interactions électroniques et la fonction que nous attribuons à la parole.

\footnotetext{
11. GOODY, 1979.

12. ONG, 1982.

13. Dans un groupe sans écriture, la loi et la mémoire sont déposées dans la parole transmise entre générations, ce qui impose que la parole demeure fidèle à ce qui lui a été confié. Cette fidélité n'est pas à considérer dans le sens d'une reproduction à l'identique des mots, comme pour l'écrit, mais comme un esprit qui est conservé au fil des énonciations successives. Le groupe se structure parce qu'il va donner à la parole circulant en son sein une valeur d'impersonnalité. Cette parole ne devra, et ne pourra pas, faire l'objet d'une appropriation personnelle par des individus particuliers sans risquer de mettre en cause ce qui relie le groupe.

14. Sur ces rapports, qui renvoient au couple Socrate/Platon, voir DERRIDA, 1980.
} 
Ce qu'il est intéressant de repérer dans notre contexte d'oralité secondaire, c'est la manière dont l'oralité est définie par rapport à l'écriture, dans un lien de dépendance par rapport à elle. Autrement dit, comment définissonsnous l'oralité dans un monde où celle-ci n'a plus la fonction primordiale d'être l'unique porteuse du sens, de donner à une communauté humaine sa cohésion ? L'oralité renvoie alors à l'idée d'échange spontané, informel, collectif. En fait, les personnes appartenant à une-culture orale considèrent au contraire que l'échange oral doit être formel ${ }^{15}$. De plus, sa dimension collective existe de fait dans une civilisation de l'oral parce que les personnes ont peu d'occasions de centrer leur attention sur elles-mêmes. Pour nous, au contraire, si l'oralité renvoie à l'idée de collectivité, c'est justement parce que cette dimension collective de la vie quotidienne a disparu. Elle représente pour nous quelque chose qui n'existe pas dans notre réalité sociale. Enfin, la spontanéité de l'échange oral existe dans une culture d'oralité primaire parce qu'il n'y a pas de support écrit permettant une prise de distance critique et réflexive. Il en est tout autrement pour notre civilisation de l'écrit.

Ces propriétés sont celles que nous attribuons à l'oralité parce que nous sommes dans une civilisation de l'écriture. L'échange oral se définit à partir d'elles parce que nous sommes conscients des effets de clôture de l'écriture. Ceux-ci se manifestent à travers la fixation d'une forme définitive, l'autorité du texte et l'absence mutuelle de l'auteur et du lecteur qui interdit d'engager le dialogue, et enfin par les décalages dans le temps et l'espace qu'induit le texte. Cependant, en même temps, l'écriture permet un sens critique, une réflexivité, une richesse polysémique, une pérennité, elle rend possible une analyse systématique des mots et des significations. Ce sont autant de propriétés que ne possède pas la parole, qui est toujours une performance d'un sujet.

On voit bien que ce que désigne l'oralité pour nous ne signifie pas la même chose que pour un groupe dans lequel l'échange oral est le seul moyen de partager, maintenir et transmettre le savoir et la culture de ce groupe. La spontanéité, le caractère informel des interactions et la participation à un collectif sont construits de toutes parts dans notre monde de l'écrit. Mon hypothèse est que le développement des communautés "virtuelles" répond à cette perte du sens du collectif tel qu'il est défini par les fonctions de la parole (et ce, même s'il y a des tentatives de la part des participants pour

${ }^{15}$. ONG, op. cit. p. 132 sq. 
compenser cette perte, comme nous le verrons dans l'analyse du débat STS). L'écriture que je qualifie de quasi-orale est une écriture qui cherche à retrouver cette capacité de lien de la parole. Elle constitue une tentative que mettent en œuvre les membres des communautés virtuelles pour faire exister, justement, ce sentiment de communauté.

Puisque l'existence du sens de la communauté reste problématique dans le cas des (injustement dénommées ?) communautés sur Internet, puisque l'expérience commune n'existe qu'à travers une pratique d'écriture et de lecture, puisque l'écriture et la lecture sont toujours des productions individuelles et solitaires, il faut bien alors que les individus construisent à travers leur écriture des indices témoignant de l'existence de cette communauté. Ce travail sur l'écriture, je l'appelle quasi-oralité. Il renvoie aussi bien aux tentatives de construction d'un sens commun qui donne au groupe des référentiels partagés, qu'à l'élaboration commune des enjeux des échanges en cours, ainsi que des procédures appropriées pour aboutir à un résultat dans un débat électronique (dont la finalité est elle-même objet de discussion). Dans l'interaction électronique, il n'y a pas de référent commun construit à travers le vécu quotidien du groupe et à travers des pratiques sociales constamment ajustées. Ce référent commun se construit habituellement à travers la parole échangée quotidiennement, ainsi qu'au travers de multiples gestes, mimiques et attitudes produites à l'attention d'autrui. Par conséquent, les participants aux échanges électroniques doivent, pour tenter de construire un sentiment de communauté, ajuster en permanence leurs propos en fonction des lectures possibles pour indiquer une intention de participation à une élaboration collective, en préciser les intentions, tout en cherchant à donner un caractère spontané à leur écrit. Ils doivent accepter de se prêter au jeu de l'échange au risque, nous le verrons, de voir leur texte repris et reformulé selon un sens qui n'était pas celui de l'auteur. Participation à une élaboration collective, spontanéité, échange, autant de propriétés attribuées habituellement à l'oralité et qui caractérisent également l'écriture - quasi orale - dans les débats électroniques. Les deux analyses de débats électroniques détaillées plus loin illustreront et expliciteront cette dynamique.

Outre la volonté de donner corps à une communauté virtuelle, ce qui peut motiver une telle utilisation de l'écriture est le sentiment d'aboutir à un consensus. Nous verrons en effet qu'il s'agit là d'une façon commode de créer un support commun qui puisse servir de base consensuelle à une communauté qui dans la réalité ne possède pas une pareille base commune. 
L'ensemble des messages qui forment ce débat électronique constitue un texte consensuel idéal, puisque toutes les positions y sont exprimées en théorie et que tout y est écrit et disponible. C'est en effet davantage la pluralité qui caractérise la communauté STS étudiée ici. Ce débat électronique peut dès lors être considéré comme un moyen de créer artificiellement une position médiane au sein de la communauté réelle, à partir du sentiment de communauté construit sur Internet et comme un moyen de fonder cette position sur l'apparence d'une participation générale des membres de la communauté à cette élaboration. On voit qu'une communauté virtuelle, bien plus qu'elle ne suscite le maintien d'un lien social, peut être un outil politique d'argumentation. Par l'intermédiaire d'une écriture quasi orale, un consensus peut ainsi être posé, sans autre forme de vérification, pour être utilisé à ce titre. Cette initiative consistant à former une représentation unifiée de la communauté STS a donc été un moyen, pour certains participants, de construire un consensus autour de leur position. En d'autres termes, cela revient à utiliser l'écriture électronique et ses propriétés manipulatoires, dans une situation définie à travers ce que suggère l'oralité pour nous.

\section{Quasi-oralité et vraisemblable}

Les participants au débat électronique ne peuvent rendre compte de la communauté STS qu'à travers l'écriture. Si, sous sa forme quasi-orale, elle tente de recréer un sens de la communauté dans l'espace de discussion virtuel, il y a une limite à cette mise en scène : les messages électroniques ne permettent pas de se repérer par rapport à un référent stable que désignerait le discours. Il s'agit là d'un problème commun à tout texte, mais il apparaît de manière d'autant plus flagrante pour l'écriture quasi-orale que celle-ci cherche justement à construire un sens commun et un sentiment de communauté. Or, ce sens commun ne peut se construire sans un recours implicite et constant à un référent dans la réalité, donc identique pour tous. La construction et le partage d'un tel référentiel commun (un référent externe) correspondent à toute une partie de l'activité quotidienne d'acteurs qui se côtoient. Cette construction ne peut pas, bien entendu, se faire à l'écrit. Cependant, elle reste implicite et son absence n'apparaît que dans certains cas, comme dans le discours ironique, par exemple $\mathrm{e}^{[6]}$.

\footnotetext{
16. Voir AYCOCK et BUCHIGNANI, 1995, pp. 184-232. Cet article rend compte de cette tension entre une intention de rendre compte d'une réalité et un risque de dérive ironique. Il analyse un débat électronique qui avait pour objet les agissements d'un universitaire canadien
} 
Une comparaison avec le texte littéraire peut nous éclairer sur le problème que pose le texte électronique. L'atmosphère d'un roman aura, pour un lecteur, des traits pris de sa réalité vécue au quotidien, mais l'évocation sera cependant différente pour un autre lecteur. Chacun construit en effet des significations à travers une fonction de représentation, sans qu'il puisse s'accorder avec l'auteur sur un référent externe. Le référent demeure interne au texte, mais sans pour autant soulever de problème particulier. Simplement, la perte de la référence socioculturelle de l'auteur, qui donne un sens local à des propos, condamne le texte à n'exister que dans la conscience des lecteurs.

En revanche, alors qu'un texte littéraire engage le lecteur dans la construction d'un monde analogue à la réalité, qui n'en reste pas moins une construction individuelle et non partageable, le texte électronique prétend se soutenir d'un référent externe. Le débat STS le montre bien, nous le verrons, en rendant compte d'une telle tension. Ainsi, par exemple, ce message en aparté d'un participant à un autre: "Ce type est vraiment sérieux, ou plaisante-t-il ?" pointe bien cette ambiguïté des messages qui peut ressortir à tout moment. Dans ce cas, le doute sur le partage d'un même niveau de lecture du texte et d'une même référence apparaît alors directement aux lecteurs.

Ainsi, les débats électroniques analysés ici mêlaient les textes de commentaires avec un contexte "réel". Il se produit dans ce cas une superposition des messages électroniques qui concerne à la fois des prises de positions diverses, des interprétations contradictoires et des éléments du contexte local expérimenté par chaque participant au débat. Une telle juxtaposition de textes produit une mise en question de chaque fragment de texte, en fonction de l'ensemble constitué par la mise en présence de ces fragments. La notion de quasi-oralité développée ici indique les problèmes que peut poser une telle juxtaposition de textes et une superposition des points de vue, des types de discours et des types de faits relatés, ainsi que les moyens mis en œuvre pour tenter d'y remédier.

Ce genre de situation ne fait que rendre explicite ce manque au niveau d'un référent externe. Elle concourt à révéler la texture même de l'écrit

(Valery Fabrikant) ayant pris en otage et tué quatre de ses collègues, au moment où se passaient les faits. Les personnes de l'université concernées par l'affaire étaient impliquées dans ce débat. 
électronique qui se présente au lecteur. Le sentiment de communauté ne peut qu'être mis encore davantage en défaut par rapport à l'idéal d'une communication collective et transparente. Alors que, pour un texte classique, la trame narrative constitue un soutien pour le lecteur afin qu'il construise une représentation de ce qui est désigné - même s'il n'y a pas de référent externe - le texte électronique, par l'effet de la superposition d'éléments disparates, prétendant appartenir à la réalité, ne peut plus se soutenir d'une telle construction. Cette absence de trame fait alors apparaître avec d'autant plus de clarté l'incohérence du discours.

Mais il reste que l'écriture, même quasi-orale, n'est pas concernée par le réel. En littérature, même le roman réaliste montre en fin de compte un ensemble de "détails concrets" dont le but est non pas de désigner des objets de la réalité, mais de signifier au lecteur qu'il se trouve devant une description réaliste ${ }^{1}$. Ce sont moins les éléments effectivement dénotés que l'activité de dénotation qui est mise en avant au travers de cette écriture. Il y a du vraisemblable dans certaines écritures littéraires, au sens où l'ont défini un ensemble de critiques et de théoriciens de la littérature : le vraisemblable met en relation non pas avec le réel mais avec ce que dit l'opinion publique du réel. C'est une mise en conformité avec un discours, non pas avec un référent ${ }^{18}$. Un phénomène similaire se produit pour l'écriture électronique quasi orale. Parce qu'un référent n'y est pas identifiable dans le réel, toute une machinerie matérielle et discursive se déploie pour nous faire croire au réel qui est censé y être désigné. Ce que j'entends donc par quasi-oralité est cet ensemble à la fois discursif et technique qui se déploie, comme dans la narration romanesque (utilisation du discours commun et techniques du récit réaliste), pour faire adhérer le lecteur à la vraisemblance de ce qui est désigné, et pour lui faire oublier qu'il s'agit d'une écriture produisant ses effets.

Cette perte d'un référentiel fixe pour le discours, ainsi que la perte du sens d'un collectif que nous avons identifiées à travers l'écriture électronique produit une incohérence du discours et d'une identification problématique à un collectif. Face à ces difficultés et aux limites de l'interaction électronique, deux positions ont été adoptées dans le cas du débat STS examiné ci-dessous : d'abord une volonté de remédier à ces limites, puis une tentative de construire un sentiment de communauté à travers une mise en

${ }^{17}$. BARTHES, 1968, pp. 84-89.

${ }^{18}$. TODOROV, 1968. 
scène de l'écriture pour le collectif, opposée à une recherche de pouvoir dans le débat à travers une utilisation de l'ambiguité induite par l'écriture quasi-orale.

Ainsi, l'utilisation de l'écriture quasi-orale - ce masque posé sur l'écriture pour faire adhérer le lecteur au vraisemblable qui y est désigné correspond-il à un choix quant à l'optique à donner aux échanges électroniques, un choix qui renvoie certes ici aux registres de l'échange oral, mais qui peut masquer un autre choix possible, celui d'utiliser au contraire à plein l'écriture et la manipulation des significations qu'elle permet, sous couvert d'oralité et de participation collective. Ces effets ont été utilisés stratégiquement par certains participants au débat STS, comme nous allons le voir à présent, en analysant successivement le sentiment de communauté qui a pu émerger du débat, sa dynamique (qui est celle de l'écriture quasiorale), et les stratégies de manipulation propres à l'écriture qui se sont développées dans le même temps.

\section{LA CONSTRUCTION DU SENTIMENT DE COMMUNAUTE DANS LE DÉBAT ÉLECTRONIQUE}

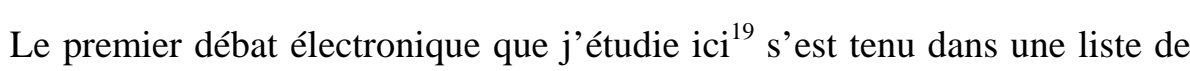
discussion identifiée sous le nom "sci-tech-society", qui était, au moment du débat, abritée par l'université de Californie à San Diego 20 . De nombreux membres de la communauté STS souscrivent à ce forum de discussions. L'origine multidisciplinaire des participants (sociologues, anthropologues, historiens des sciences, mais également scientifiques, ingénieurs, administrateurs de la recherche et économistes) a constitué un terrain favorable pour lancer le dialogue autgur de questions qui les préoccupaient à partir de leurs positions respectives ${ }^{21}$.

\footnotetext{
19. L'analyse du débat STS est, en partie, une reprise de HERT, 1997.

${ }^{20}$. L'adresse de la liste est : listserv@kasey.umkc.edu.

${ }^{21}$. De nombreux membres de cette liste de discussion sont des membres reconnus de la communauté STS internationale. Les membres proviennent de 24 pays, les deux tiers étant Américains (65\%) et une minorité des autres pays (le Canada avec $7 \%$ et l'Angleterre avec $6 \%$ étaient aux deuxième et troisième rangs). Seule une faible minorité de chercheurs non américains ont participé au débat électronique. Sachant que ses enjeux se référaient en général au contexte américain de la recherche, cette orientation a pu décourager ou contrarier les membres de la liste provenant d'autres pays.
} 
Le débat STS débuta le 3 octobre 1994 par un message de Patrick W. Hamlett qui portait le titre suivant: "STS est attaquée2 ". Ce message était une réaction à un article paru dans la revue Science datée du 9 septembre qui avait pour titre "Science is under attack ${ }^{23}$ ?". Cet article considérait les récents développements théoriques dans la communauté STS, en particulier le constructivisme social, comme une attaque contre la "Science et la Raison ". L'article témoignait en fait d'un manque d'informations et de précisions au sein même des communautés scientifiques sur les perspectives théoriques investies par la communauté STS. Le débat électronique se termina le 12 décembre, après que 230 messages concernant uniquement ce débat eurent été envoyés. Une rencontre à l'université de Durham (Angleterre) organisée les 3 et 4 décembre 1994 par Steve Fuller, l'un des participants les plus actifs du débat, sur le même thème que le débat électronique, compléta le débat en ligne. Les conclusions de cette rencontre furent présentées dans la liste de discussions du 9 au 12 décembre. Le journal Times Higher Education Supplement, à la suite d'une proposition de Fuller, suivit le débat électronique durant ces 4 jours et publia les messages les plus significatifs. En outre, ce débat constitua les prémices d'une controverse plus importante qui a mobilisé une partie du monde académique français en 1998, ce que l'on a appelé l'affaire Sokal ${ }^{24}$.

Nous verrons dans cette partie comment un sentiment de communauté a été construit au fil des échanges. En partant de l'impact réel de la communauté des participants au débat, nous verrons les tentatives employées pour construire un tel sentiment. Elles reposent sur la politique de mobilisation visible dans le contenu de certains messages. La mise en évidence d'un contexte plus large, c'est-à-dire celui de la communauté STS et non de la communauté électronique, permettra ensuite d'éclairer plus précisément

\footnotetext{
22. Les échanges dans la liste STS se faisant en anglais, tous les textes des messages concernant le débat ont été directement traduits de l'anglais par l'auteur.

23. "La science est-elle attaquée ?" (page 1508).

${ }^{24}$. Voir SOKAL et BRICMONT, 1997. Alan Sokal, physicien américain renommé, a voulu s'attaquer à travers son canular (un vrai-faux article envoyé à la revue Social Text et publié par elle) à la communauté des sciences humaines, et en particulier aux courants postmodernes et STS. Cette mauvaise blague était destinée à montrer que l'on peut publier dans ces domaines des textes qui ne font qu'imiter un style de discours sans pour autant livrer un contenu sérieux, puisque dans un second article, Sokal a avoué la supercherie. Pour une analyse détaillée des enjeux de cette controverse pour le champ STS et quant aux rapports entre domaines scientifiques, voir JURDANT, 1998 et JEANNERET, 1998.
} 
l'implication effective dans le débat et de conclure sur la portée de ce sentiment de communauté.

La partie suivante montrera les différentes stratégies utilisées pour développer le débat. Ces stratégies, mais aussi ces tactiques, renvoient respectivement aux registres de l'écrit et de l'oral. Enfin, dans une troisième partie, l'analyse des échanges dans un débat entre chimistes nous permettra de mettre en perspective les usages d'une liste qui situe les enjeux des échanges au niveau d'une communauté internationale avec ceux d'un espace de débat local. Ces deux niveaux complémentaires fournissent un cadre d'interprétation de la construction, des possibilités, des limites d'un espace de dialogue à travers ces dispositifs électroniques de communication.

\section{La communauté des participants}

Il n'y a pas une simple reproduction d'un type de communication (les controverses et les débats scientifiques dans notre cas) dans un média différent lorsque les utilisateurs se l'approprient ${ }^{25}$. De manière générale, les utilisateurs d'une technologie la rencontrent toujours dans un contexte particulier qui influence en retour leur compréhension de cette technologie ${ }^{26}$. Il apparaît qu'une activité créative est mise en œuvre lorsqu'il s'agit de s'approprier le média, tout en conservant un cadre ancien et habituel qui caractérise l'expression du discours académique. Cette souplesse permet à des utilisateurs de tirer parti du dispositif de communication pour en compenser les limites et influencer leur communauté. Baym ${ }^{27}$, en se fondant sur de précédentes études sur la communication médiatisée par ordinateur (Computer-Mediated Communication), a développé l'idée selon laquelle les participants à un groupe de discussion électronique utilisent créativement les fonctionnalités du système pour établir une dynamique sociale. Ainsi, selon cet auteur, les participants à un débat électronique compensent activement ces limitations sur le plan des indices sociaux, à travers les interactions dans lesquels ils s'engagent. Selon Baym, puisque le média n'assigne pas des formes rigides aux interactions qui ont lieu, il reste des marges de manœuvre pour véhiculer un sens social à travers elles. Cette dynamique sociale porte sur l'élaboration de formes d'expression, la création de rapports sociaux

\footnotetext{
25. ORLIKOWSKI et YATES, 1992.

26. WOOLGAR, 1987.

27. BAYM, 1995.
} 
improbables dans d'autres situations, l'élaboration de normes de conduite et l'exploration d'identités réelles ou fictives ${ }^{28}$. Ces pratiques permettraient aux participants à des forums électroniques d'inventer de nouvelles communautés, fictives. Cependant, une telle notion de communauté demeure uniquement fondée sur les règles de communication que les participants aux échanges inventent : elle reste donc une entité formelle et problématique, et ce, même lorsqu'elle est accompagnée d'une communauté réelle.

Environ $16 \%$ des membres de la liste ont participé au débat (75 parmi 450 inscrits sur cette liste au moment du débat, bien qu'il n'ait pas été possible de suivre le mouvement d'inscriptions et de désinscriptions à la liste). Ce faible taux de participation nous renvoie ici d'autant plus à la question de la légitimité de parler de "communauté" de participants à propos de ces interactions électroniques. McLaughlin, Osborne \& Smith ${ }^{29}$ ont étudié l'usage de la métaphore de la "communauté" dans le cadre des interactions électroniques. Leur argument est que la forte proportion de membres de la liste qui n'envoient pas de messages (appelés lurkers en anglais) dans tout groupe de discussion électronique rend problématique l'existence effective d'une communauté. Selon ces auteurs, les communautés qui se réclament de tels groupes de discussion sont amorphes et éphémères. Les mêmes restrictions s'appliquent à la situation que j'analyse ici. Cependant, l'existence d'une réelle communauté sociale se réclamant du mouvement STS, avec ses rencontres périodiques, ses colloques et ses revues, rend plus complexe l'interprétation. Dans un tel cas, le média n'est pas en effet sensé créer une communauté, c'est une communauté existante qui utilise le média. De plus, l'étude de l'origine des messages montre que $17 \%$ (13 parmi 75) des participants ont envoyé environ $50 \%$ (102 parmi 230) des messages, et que près de la moitié des participants (43\%) n'ont envoyé qu'un seul message durant le débat. Ainsi, la portée de la notion de sentiment de communauté émergeant de cette liste de discussion reste bien hypothétique.

La liste STS a été créée, selon son gestionnaire, dans un souci de construire une communauté STS d'étudiants et de favoriser les échanges d'idées à propos de ce que devrait ou pourrait être une communauté intellectuelle interdisciplinaire. Or, chercheurs et enseignants STS se sont rapidement

\footnotetext{
28. On peut noter que les usages des messageries du Minitel en France correspondent largement à des phénomènes similaires. Josiane Jouët a notamment exploré les aspects de la construction d'identités fictives dans ces forums électroniques. JOUET, 1990.

29. McLAUGHLIN, OSBORNE, SMITH, 1995.
} 
intéressés à ce forum qui est alors devenu un espace d'échange pour toute la communauté STS, et ce bien avant le développement du débat analysé ici. En définitive, plus aucun étudiant n'a participé aux échanges ou envoyé des demandes de renseignements. Cette dérive de la liste par rapport à son intention initiale indique qu'une prétendue communauté électronique reste bien peu consistante lorsque les intérêts stratégiques d'une communauté réelle l'entraînent à coloniser cet espace de discussion. Cependant, pour parvenir à réaliser cette colonisation, il n'est pas suffisant d'être une communauté déjà constituée. Il faut être en état de mobiliser les membres et de créer une dynamique collective. Voyons, compte tenu de la faible proportion de participants au débat, quels moyens ont été utilisés pour susciter cette mobilisation.

\section{La mobilisation d'une communauté}

Si nous examinons le contenu des messages, il apparaît que les enjeux principaux du débat concernaient précisément le rôle de la communauté STS. Outre le caractère très mobilisateur d'une telle thématique, du fait qu'elle touchait aux fondements de la communauté STS, celle-ci a également servi à développer le sens de la communauté dans l'espace virtuel du débat. Le débat faisait référence à plusieurs points stratégiques qui animent la discussion dans le champ STS, ce qui a probablement conduit un certain nombre de chercheurs à se lancer dans la discussion ${ }^{20}$. Ces points étaient suffisamment problématiques pour susciter de nombreuses réactions, lesquelles ont révélé la grande hétérogénéité des points de vue qui existait au sein du mouvement STS.

Le livre écrit par Paul Gross et Norman Levitt ${ }^{31}$ a souvent été cité pendant le débat. Cet ouvrage est une critique en règle de l'analyse constructiviste de la science. Gross participa au débat dans la liste de discussion. Il rejeta vigoureusement la légitimité de toute étude STS portant sur l'activité scientifique, ce qui provoqua de vives réactions. La question du respect du travail des chercheurs dans le domaine STS par d'autres chercheurs fut un

\footnotetext{
30. Des aspects polémiques ont été soulevés, comme le rôle de la communauté dans des questions de politique scientifique, ou encore les raisons qui poussent les scientifiques à refuser une légitimité aux travaux STS. A partir de ces questions est apparu un enjeu particulier: celui de montrer aux yeux des scientifiques et du public l'importance des recherches dans le domaine STS.

31. GROSS, LEVITT, 1994.
} 
point critique. Il en résulta une question posée à l'ensemble de la communauté : "Que tentons-nous vraiment d'accomplir dans la durée ?". De nombreuses réponses à cette question furent formulées sous la forme de descriptions de projets individuels. Ainsi, bien des aspects de la communauté STS - ou du réseau STS comme l'ont formulé certains participants - ont pu être ainsi présentés. L'hétérogénéité des pratiques, des enjeux, des buts et des idées relatives à l'idée fédératrice de la construction sociale des sciences en a été rendue manifeste.

Pour des raisons techniques, il n'était pas possible de connaître la liste effective des personnes abonnées à la conférence électronique. La conséquence de cette incertitude a été que les membres de la liste de discussion avaient une perception très vague de ceux qui étaient les destinataires effectifs des messages et des revendications. Parfois, la discussion se transformait en un appel à la mobilisation de la communauté STS, en réponse à "l'attaque" qu'elle était en train de subir, comme l'indique le message suivant :

Date: Wed, 5 Oct. 1994 ; Subject: STS est attaqué - à nouveau !

“(...) Nous avons été mis au courant et il est temps de nous présenter au monde à la manière dont nous nous situons nous-mêmes plutôt que de laisser les autres nous définir, définir nos buts et nos perspectives (...)"

Ces incitations constituaient des tentatives d'utilisation de la liste de discussion pour rassembler les diverses orientations qui forment la communauté STS et former ainsi pour elle une perspective d'ensemble. Il est révélateur pour notre propos de constater que ce genre de thématique est apparu précisément dans cette liste de discussion, et non lors d'une manifestation plus conventionnelle du type colloque. Cet aspect met en avant, en effet, le sentiment d'existence d'une communauté accessible directement par l'intermédiaire du support informatique. Pour mettre en œuvre les capacités de ce média et mobiliser la communauté virtuellement présente, plusieurs participants ont développé l'argument selon lequel face à une "attaque" il s'agissait d'adopter une démarche cohérente et de dénoncer les représentations erronées de la communauté qui sont diffusées par ses ennemis. Ces "ennemis" (hypothétiques) sont en fait un moyen efficace pour fédérer un groupe disséminé. Argumenter en faveur de l'urgence d'une action pour défendre la position du mouvement STS à l'égard de la science (malgré la grande diversité de points de vue qui coexistent), offre un moyen de construire un sentiment de communauté à 
travers le média électronique. Ce dernier est alors mis à la place de l'agent matériel qui va rendre possible la convergence des perspectives.

L'idée implicite défendue par les participants est ici la suivante : si toute la communauté peut s'exprimer à travers ce moyen et si une question capitale pour cette communauté est soulevée, alors il devient possible, grâce à ce média, de construire une position synthétique à partir de l'ensemble des interactions. Mais, même si cet argument pouvait avoir une pertinence par rapport au type d'interactions qui ont lieu dans un forum électronique, celui-ci est fondé sur le présupposé que toute la communauté STS - ou du moins une part significative de celle-ci - peut être mobilisée par ce moyen. Or rien n'est moins sûr, puisque aucun participant n'était en mesure de connaître la diffusion réelle de cette liste dans la communauté, si ce n'est à l'aide des sondages que chacun pouvait faire en direction de ses collègues. Le sentiment de communauté issu des interactions dans la liste de discussion repose donc sur le lien que la machine est supposée établir entre les membres de la communauté STS.

\section{La construction de l'implication dans le débat}

Si certains messages émis durant le débat ont tenté de créer une mobilisation collective et donc un sentiment d'existence d'une communauté susceptible de réagir à tout moment par écran d'ordinateur interposé, l'implication effective des participants au débat a suivi plusieurs voies. Ainsi, comme nous allons le voir, malgré la continuité qui s'était établie au sein du débat, l'implication dans ce débat a généralement suivi d'autres chemins que celui du virtuel.

Les deux périodes de croissance importante du débat correspondent à des phases où certaines remarques ou questions ont suscité de nombreuses réactions, en particulier de la part de nouveaux participants. Les questions et les réactions qui ont contribué à une mobilisation importante durant ces deux périodes peuvent être résumées sous les deux en-têtes de messages suivants : "STS under attack" ("STS est attaqué ") et "You don't get no respect" ("On n'est pas respecté"). Ces deux en-têtes ont souvent été réutilisés puisque de nombreuses interfaces de messageries ajoutent automatiquement le même en-tête lorsque l'on répond à un message. Ainsi, 26 messages ont repris le premier en-tête et 88 le second. L'utilisation du même en-tête le long des différents messages qui se succédaient a rendu 
ainsi visible une certaine cohérence dans le débat. Cette utilisation est ainsi une manière purement écrite de construire une continuité dans les échanges, mais également de donner à voir au lecteur l'importance d'un thème dans le déroulement d'ensemble du débat. C'est bien là une manière de réinscrire dans le texte des éléments de la participation collective, et de lutter ainsi contre l'écriture, toujours hors contexte, et qui abolit la dimension collective, spatialement et temporellement située.

Toutefois, cette inscription dans la continuité ne s'est pas soldée par une véritable pérennité des échanges. La mobilisation a été forte durant les premiers jours du débaf ainsi que trois semaines plus tard, à partir du vingtième jour environ ${ }^{2} 2$ La croissance du nombre de nouveaux participants s'est ensuite progressivement estompée, jusqu'à ce que le débat cesse brutalement, au bout de 75 jours. Cet arrêt brusque, alors que les questions posées n'étaient pas tranchées, indique bien le caractère éphémère de la mobilisation, et le caractère hypothétique d'une communauté électronique, alors même qu'une communauté réelle utilise le dispositif. Cet abandon soudain du débat électronique peut s'expliquer par le fait, comme nous allons le voir ci-dessous, d'une reprise du débat par d'autres moyens: articles, colloques, échanges de messages électroniques privés. Il indique que, contrairement à l'idée d'une possibilité permanente de débattre sur tous les sujets imaginables, les forums de discussions électronique ne sont pas ces espaces idéaux de l'échange direct, immédiat, transparent. D'autres modes de communication, plus classiques, ont en l'occurrence été préférés pour la poursuite du débat.

La tendance des membres de la liste à échanger des points de vue sur le débat, suivant d'autres voies, (rencontres, échanges téléphoniques, épistolaires, ou plus simplement échanges de courriers électroniques privés) est un élément qu'il faut donc également prendre en considération ici. Ces échanges peuvent être évalués à travers les réponses à un questionnaire que j'ai envoyé aux membres de la liste ${ }^{\frac{3}{3}}$. Certains membres de la liste ont

\footnotetext{
${ }^{32}$. Le nombre de participants se rajoutant à la discussion a évolué rapidement au début pour ralentir et stagner ensuite: on constate qu'après le premier mois, $80 \%$ des participants avaient envoyé au moins un message.

${ }^{33}$. J'ai reçu 23 réponses à mon questionnaire. Elles provenaient de participants et de nonparticipants au débat. Mes questions étaient les suivantes :

- Avez-vous échangé des messages privés concernant le débat? Si oui, avec des membres de la liste?

- Quelle est votre conclusion sur le débat, à la fois sur le plan de la forme et du contenu ?
} 
interagi à travers des messages privés pour confirmer leurs positions ou demander des précisions par rapport au débat. Ces réponses constituent la part invisible du débat. En ce sens, le débat public sur la liste électronique n'a été que la partie émergée de l'iceberg. La proportion d'interactions privées ayant trait au débat n'est pas véritablement évaluable. Pour avoir une idée de cette communication "masquée", on peut se référer au témoignage qu'un participant m'a envoyé après l'arrêt du débat :

“ Il y a eu 65 messages (surtout d'Américains, mais également de quatre pays Européens) dans mon fichier de courrier électronique à propos de cette discussion; environ dix étaient des messages destinés à la liste (y compris les miens) et tous les autres étaient des messages 'privés' portant sur mes messages. J'ai entendu d'autres personnes pour lesquelles cette proportion correspond à leur expérience.

De ces 55 messages 'privés', 25 environ étaient des messages de cinq personnes, qui ont poursuivi pendant une semaine environ leurs commentaires à propos des messages 'publics' qui continuaient à être envoyés. (Les cinq comprenaient deux étudiants de troisième cycle, un assistant de professeur, un professeur associé et un professeur ; tous, sauf un, étaient Américains. Je connaissais assez bien deux d'entre eux, les autres étaient à ce moment des connaissances).

La plupart des messages 'privés' émanaient de personnes qui envoient rarement des messages 'publics' à la liste, pour autant que je sache."

Ainsi, le dispositif permet-il tous les apartés possibles, sans que les participants ne s'en rendent compte, trahissant ainsi l'intention de faire exister une communauté. Alors que dans l'échange oral, il n'est pas possible de faire des apartés sans être remarqué par le reste de l'auditoire ou par celui qui s'exprime, ces pratiques sont passées totalement inaperçues aux autres

- Pensez-vous que ces formes d'échanges sont une nouvelle manière de débattre de questions concernant la communauté STS dans son ensemble ? Pensez-vous que ce moyen complète les colloques?

- Pensez-vous que ce mode d'interaction est plus démocratique qu'une conférence classique ? - Pensez-vous que ce mode de communication est utilisé différemment dans d'autres domaines scientifiques, non transdisciplinaires ?

- Etes-vous satisfait par la tournure du débat (de nombreuses positions, mais peu de conclusions)?

- Si vous avez participé au débat, pourquoi avez-vous choisi de le faire ? Que pensez-vous des propositions faites durant le débat?

- Si vous n'avez pas participé, quelle a été votre position sur le débat?

- Quels sont vos domaines d'intérêt? 
participants. De plus, il semble que ce soit précisément ces échanges "privés" qui aient été considérés comme les plus intéressants, d'après le commentaire suivant, parce qu'ils ont servi en quelque sorte d'introduction, à des échanges - oraux et en dehors d'Internet - entre participants. Ces échanges privés ont permis de poursuivre le débat en dehors de la liste. De même, ce débat a été repris dans plusieurs sessions au cours du colloque annuel de la société des études sociales sur la science (Society for the Social Studies of Sciences - 4S) de 1995. Ce colloque est le lieu de rencontre des chercheurs STS de tous les pays, et a été l'occasion de formuler différemment les questions soulevées dans un premier temps dans la liste électronique.

“Le colloque $4 S$ suivant a été très intéressant pour moi, tout d'abord à cause de toutes mes discussions avec les personnes qui m'avaient envoyé ces messages 'privés' et, en second lieu, à cause de toutes les discussions avec des personnes qui ont lu les échanges mais qui ne se sentaient pas à l'aise pour livrer leurs pensées par voie électronique. La plupart de ces rencontres en 'face à face' étaient substantielles et intéressantes à mes yeux ${ }^{34}$.",

Tandis que le débat avait cessé depuis 10 mois dans la liste de discussion, l'importance, au sein du colloque $4 \mathrm{~S}$, des questions soulevées auparavant dans la liste électronique illustre la continuité de l'intérêt de la communauté pour ces questions. Le débat n'était effectivement pas clos et on peut légitimement supposer que le colloque a été un moyen d'aborder ces questions d'une manière que la liste électronique ne permettait pas : c'est-àdire par une rencontre directe, orale. Ainsi, si nous pouvons simplement suggérer ici que le débat électronique a constitué un prémisse à des échanges et rencontres de membres de la communauté STS, lors du colloque $4 \mathrm{~S}$ notamment, il est cependant peu vraisemblable que le débat électronique seul ait été un moyen pour des individus de la communauté STS de resserrer leurs liens hors de leur cercle de connaissances déjà établi ${ }^{\mathrm{B} 5}$.

\footnotetext{
34. Entretien de l'auteur avec avec Sharon Traweek, le 10 octobre 1996.

35. Freeman (FREEMAN, 1984) a constitué un cadre théorique pour caractériser la structure sociale d'une communauté scientifique en rapport avec les liens entre scientifiques. Il a utilisé la notion de conscience de l'existence d'un collègue (awareness) en opposition avec la notion de connaissance d'une personne (aquaintaceship), pour distinguer deux types de relations fondamentales qui relient les acteurs d'un projet ou d'une communauté. L'auteur considère que l'émergence d'une spécialité scientifique est liée au passage de la conscience de l'existence d'un pair à la connaissance et la proximité avec celui-ci. Ce passage est une condition nécessaire (mais non suffisante) au développement d'une communauté scientifique.
} 
Pour résumer, on peut dire, comme l'a remarqué un participant en réponse à mon questionnaire, “qu'il était intéressant de voir ce que pensent certaines personnes". Et d'ajouter: "J'ai toujours trouvé les échanges personnels [privés] les plus intéressants". Enfin, une autre réaction a été que "le débat n'a pas abouti à un consensus, mais que chacun a tiré ses conclusions". Ainsi, les positions dans le débat ont été jouées pour les membres silencieux de l'audience. De plus, comme il n'a pas été possible de connaître le nom des personnes qui ont lu les messages de la liste sans s'y manifester (les lurkers), les participants à la " communauté " électronique n'en connaissant pas les membres. Ainsi, les messages échangés au cours de ce débat, loin de constituer la base d'une communauté électronique, ont relevé d'une opportunité pour certains participants, non seulement pour trouver des stratégies de stimulation et de développement du débat, mais également afin de l'utiliser en fonction de leurs intérêts.

\section{STRATÉGIES POUR DÉVELOPPER LE DÉBAT}

Dans cette partie, je vais décrire la manière dont certains participants ont utilisé l'ambiguïté entre l'écriture et l'oralité, manifestée à travers la quasioralité, pour développer le débat. $\mathrm{Si}$, comme nous l'avons montré dans la partie précédente, la notion de communauté liée à ce type de dispositif reste problématique et hypothétique, il nous faut dès lors abandonner l'hypothèse de la capacité de l'écriture électronique à mobiliser les membres d'un groupe de discussions, ainsi que celle de l'existence d'une communauté virtuelle. Par conséquent, l'écriture quasi-orale ne permet pas de faire exister un sentiment de communauté, mais sert des politiques de pouvoir. En effet, lorsque l'on parle de construction sociale, il y a un aspect d'élaboration collective et de négociation qui est impliqué, mais également une dimension de lutte pour le pouvoir et la reconnaissance. Ainsi, différents objectifs ont été poursuivis à travers les manières de construire un rapport au texte dans l'écriture. Je les identifierai en termes de tactique et de stratégie. Ces deux aspects seront illustrés à travers deux solutions mises en œuvre au cours du débat. Ces deux modalités peuvent être opposées en termes d'oralité (d'écriture quasi orale) et d'écriture (utilisation des possibilités de l'écriture), ou encore, en termes d'action tactique et d'action stratégique.

Il a également analysé l'influence des interactions électroniques pour faciliter l'émergence d'une spécialité scientifique. Sa conclusion indique leur effet très indirect. 
La première solution qui a été utilisée pour développer le débat, est liée à la pratique de réutilisation, de citation et de reprise de messages précédents concernant la discussion. Ces pratiques ont fait apparaître un sens contextuel aux messages envoyés. De plus, ce type d'écriture utilisé dans la construction du débat visait à lui conférer une fonction de lien social, c'està-dire de régler les rapports du groupe, d'en être la mémoire. En ce sens, ces pratiques peuvent être rapprochées d'interactions orales et seront identifiées à une écriture quasi-orale.

La seconde attitude a été, pour certains participants, de construire leur autorité dans le débat en en redéfinissant les enjeux et les interprétations. Elle renvoie à une utilisation des effets de l'écriture (reprise et analyse hors contexte des termes et propositions, construction d'une position synthétique rassemblant un ensemble d'autres textes), tout en utilisant éventuellement le sentiment de communauté que tente de développer l'écriture quasi-orale.

\section{Ecriture quasi orale et effet dialogique}

Le débat électronique s'est développé pour les membres de la liste de discussion en même temps qu'une compréhension de sa nature et de ses enjeux. Sa durée (deux mois et demi), le grand nombre de messages envoyés (230 concernant le débat), leur longueur moyenne de plus d'une page, la réaction rapide de certains et les références faites à d'autres messages dans leurs propres messages ont contraint les participants à suivre la discussion en détail. Il leur était en effet nécessaire de lire de nombreux messages pour comprendre le sens, mais également le contexte de la discussion. Les nombreuses interactions ont créé une mémoire collective pour les membres de la liste, et ont formé le contexte d'énonciation. On peut même dire que les nouveaux arrivants ont été socialisés dans le groupe de discussion à travers ces lectures préliminaires des messages résultant des interactions passées. Toutefois, cette pratique de lecture collective ne signifie pas pour autant qu'une communauté électronique pouvait se définir à partir d'elle. En effet, ce contexte d'énonciation ne pouvait être correctement interprété que si le lecteur partageait le savoir implicite de ceux qui avaient envoyé les messages. En d'autres termes, pour comprendre le sens du débat, le lecteur de ces messages devait être un membre de la communauté STS.

Souvent ce n'est pas seulement le contenu de textes qui aide à lier un groupe. C'est également la circulation large de l'information qui permet de 
créer une communauté. Les caractéristiques essentielles d'un document scientifique sont la mobilité et l'immutabilité36. Elles permettent la diffusion des faits scientifiques (mobilité) sans être pour autant déformées à travers des reprises successives (immutabilité). Ces groupes de discussions électroniques respectent la mobilité, mais pas l'immutabilité. La mobilité confère alors aux messages circulant dans les listes électroniques un rôle social dans la communauté scientifique dans laquelle ils circulent, même s'ils ne sont pas des documents scientifiques. Les messages électroniques sont " mutables" en ce sens qu'ils sont parfois coupés, copiés et collés de message en message. Peut-on alors dire que, grâce à cette large circulation de messages, un sens commun de ce qu'est la communauté STS a pu émerger au cours du débat pour les membres de la liste de discussion? Notre analyse de l'absence d'un référent dans les messages électroniques nous suggère le contraire. Cependant, c'est bien cet effet de large circulation des messages électroniques qui a été investi par certains participants au débat.

Si l'on examine de près cette propriété de mutabilité, on constate qu'elle engendre des pratiques de lecture des messages qui donnent une importance particulière aux premiers participants ou aux participants réguliers. Les premiers à envoyer des messages sont lus, repris et cités plus souvent. Leur position se trouve renforcée dans ce qui se constitue progressivement comme étant le cadre de la discussion (son cheminement, les questions et problèmes soulevés). Bien qu'il n'ait pas été nécessaire de lire tous les messages précédents du débat pour réagir à un message spécifique, de nombreux messages se référaient à d'autres messages, de manière directe (en citant des passages) ou implicite (en évoquant simplement le ou les messages). Il était donc nécessaire, pour tous ceux qui voulaient s'impliquer dans la discussion, de suivre l'évolution chronologique du débat à travers un enchaînement de messages formant la trame du débat.

Cette mémoire nécessaire au débat a induit la pratique, fort commune par ailleurs, de citation de messages et d'envoi à la liste de messages synthétisant un ensemble de messages de plusieurs participants. Une convention généralement admise et qui est une fonctionnalité des logiciels de lecture des messages électroniques, consiste à insérer le texte d'un message lorsque l'on y répond. Certaines interfaces de lecture de messages permettent une édition du texte original (coupures dans le texte, voire

\footnotetext{
${ }^{36}$. LATOUR, 1989.
} 
réécriture) qui est ajoutée à la réponse. Cette pratique d'ajout de messages originaux à des réponses, produit une continuité et un effet de conversation. Orlikowsky et Yates ${ }^{37}$ définissent un genre de messages électroniques, qu'ils appellent dialogique. Ce genre est semblable à un dialogue oral, mais il utilise les possibilités du média écrit pour insérer, en partie ou en totalité, les messages précédents de son interlocuteur. Ces pratiques de communication "intègrent une continuité et une interdépendance entre les messages", comme le signalent les auteurs. Cette fonctionnalité, difficile à produire à travers un support de papier, permet de faire des connexions entre messages. Il s'agit d'une manière, pour les participants, de faire connaître ce à quoi ils se réfèrent dans leurs messages. Ce genre dialogique est caractérisé par l'insertion de messages d'autrui dans ses propres messages et par un jeu de questions-réponses entre les positions de différentes personnes qui cohabitent dans le même message. Il se forme ainsi une sorte de polyphonie que l'on peut voir apparaître graphiquement (le message original dans une réponse apparaît à travers des marqueurs comme ce signe > qui le rend immédiatement identifiable et permet de faire une sorte de séparation des voix). Mais cette référence peut également se faire de manière indirecte, lorsque des participants sont cités dans un message par leur nom et à travers un résumé de leurs positions. De la pratique de la citation, on passe ici à une pratique de synthèse, et l'on verra plus loin quelles peuvent être les conséquences d'un tel passage. De manière générale, ces pratiques de citation de messages ont des influences sur la compréhension collective du débat, comme le montre l'extrait suivant:

Date: Wed, 9 Nov. 1994 ; Subject: retour sur l'attaque (long)

[A] Maintenant que les choses se sont un peu calmées, j'ai pensé amener ma petite idée. Je ne veux pas m'encombrer ici de procès d'intentions envers certaines personnes, autrement il me faudrait un tableau noir pour les mettre toutes. Il n'y a pas d'intentions de faire des affronts dans ce message.

[B] (...) Si vous devez rassembler les troupes, il est utile d'avoir un ennemi défini, et il semblerait qu'une partie de la communauté scientifique se soit emparée de la communauté STS en les identifiant comme étant des traîtres. Bien entendu, pour cela, il leur faut se concentrer sur la partie la plus radicale (et donc prête à être identifiée comme dangereuse) de ce que nous savons tous être un monde très hétérogène. Dans ce sens, leur intérêt est de maintenir le débat polarisé ; lorsque vous vous accordez sur la diversité de STS, la menace perçue ne peut que diminuer et il devient beaucoup plus difficile d'utiliser STS comme un moyen de soutenir et protéger la science.

\footnotetext{
37. ORLIKOWSKI, YATES, 1994.
} 
[C] C'est pourquoi je ne crois pas qu'il soit très utile de dépenser du temps et de l'énergie pour réfuter les différentes perceptions erronées et les critiques non fondées. En revanche, je crois que STS a besoin de travailler à se présenter comme un moyen de réfléchir à des enjeux importants qui concernent beaucoup de personnes (...)

Cet extrait illustre la multiplicité des niveaux auxquels les messages du débat pouvaient être lus. On peut distinguer ici trois niveaux.

A un premier niveau, apparaissent le contenu et les intentions du message [B] ${ }^{8.8}$. Cet extrait fait partie de l'ensemble des suggestions, pour réagir et répondre à l'attaque dont la communauté STS a été l'objet. L'auteur s'est adressé à l'ensemble de la communauté, dans son message, en voulant attirer l'attention sur l'importance de la façon dont la communauté STS était perçue en-dehors du champ.

Un second niveau [C] montre qu'une partie du texte est constituée de références tacites à des messages plus anciens et à des réactions qu'ils ont suscitées. Le sens de ce fragment de texte n'est compréhensible que dans le contexte du débat. Les commentaires concernant le niveau plus large de la discussion sont abordés à ce niveau. L'auteur du message guide celui-ci dans le réseau de textes que constitue ce débat. Le message trouve son sens dans le débat grâce au travail de son auteur pour le relier à d'autres et grâce également aux lecteurs successifs qui vont le reprendre et éventuellement le mentionner dans des messages ultérieurs qu'ils adresseront.

Le troisième niveau qui peut être distingué est celui où sont réintégrés des éléments pouvant compenser les mauvaises interprétations des messages (notamment pour un message perçu comme polémique, alors que ce n'était pas l'intention de l'auteur). Des indices sur l'intention de l'envoyeur du message doivent être réintégrés dans le message. Autrement dit, il s'agit de redonner une portée sociale aux échanges de messages électroniques pour que les interlocuteurs parviennent à en comprendre le sens. Un moyen de le faire est d'accroître les interactions, explications et commentaires sur les messages eux-mêmes [A]. Ce travail d'extension de l'écriture pour y inclure des éléments contextuels (même si cette notion de contexte est problématique), se rapporte à ce que j'appelle ici la quasi-oralité de l'écriture électronique.

\footnotetext{
38. Les lettres $[\mathrm{A}],[\mathrm{B}],[\mathrm{C}]$ ont été rajoutées par moi pour présenter les trois niveaux de lectures de ce message.
} 
Ces actions peuvent être rapprochées d'une utilisation de l'échange écrit en tentant d'y incorporer ce que permet en général l'échange oral : créer une participation collective des interlocuteurs présents à l'être de la parole, qui se définit hic et nunc dans le contexte de l'échange. C'est probablement également là une tentative pour intégrer des éléments de la réalité dans le texte : si le texte seul ne peut soutenir un discours sur la réalité, le recours à une mise en scène des jeux entre les textes sous une forme de dialogue est peut-être la réponse trouvée par les participants au débat électronique pour tenter d'y remédier.

\section{La construction de l'autorité par l'écriture}

Après ces considérations sur la construction d'un contexte commun, nous allons nous pencher sur l'émergence de "leaders" dans le débat. Certains participants ont tenté d'utiliser l'espace de discussion publique pour occuper une place centrale dans le débat. Cette pratique peut être assimilée à une construction de l'autorité de certains participants dans le débat à travers les formes d'interactions qu'ils ont adoptées. Ecrire est une manière de construire des stratégies narratives pour produire des "effets de vérité". C'est également une manière de fixer un cadre stable, permanent, qui contraint l'ensemble des significations possibles pour définir une situation. Ainsi, l'écriture utilisée dans un cadre de débat électronique est cette technique qui consiste à tenter de prendre le pouvoir dans l'échange, en utilisant les effets d'oralité qui émergent de ce type d'échange. Ces effets sont ceux qui consistent à utiliser le sentiment de communauté résultant des tentatives pour élaborer une position collective à travers l'écriture quasi orale. Par ce moyen, les participants qui voulaient occuper une place centrale dans le débat ont tenté de redéfinir cette position collective en fonction de leurs intérêts et des arguments qu'ils défendaient, et ce en utilisant le fait que tous les échanges s'effectuent par écrit. Le comportement de ces participants peut être analysé de deux façons.

Tout d'abord, la construction de leur autorité à travers la redéfinition du débat se traduit par un travail de synthèse des différentes positions exprimées dans les messages. Lorsque ces participants avaient repris les messages d'autres personnes, en les citant ou en les reformulant, cela leur permettait de comparer ces arguments avec leurs propres positions et, bien entendu, de construire leur argumentaire de sorte que leur position apparaisse centrale dans le débat. 
En second lieu, la citation des messages d'autrui a conduit à leur manipulation. Certains participants se sont plaints durant la discussion de voir leurs propres messages "disséqués" dans des messages qui étaient supposés répondre aux leurs. Les participants observaient un mouvement de désappropriation de leurs messages au fil du débat, comme le suggère le message suivant :

Date: Fri., 07 Oct. 1994 ; Subject: UNE CLARIFICATION

Il est apparemment dangereux d'écrire un message provocateur à notre liste. Il a tout d'abord été dit que je pense qu'il n'existe pas de réalité extérieure puis que je ne crois pas que l'histoire et la philosophie des sciences (ne) sont (pas ... sic) de beaucoup de valeur. Cela n'est pas non plus ce que je crois (...)

Il a également été dit que je n'estime pas ceux qui écrivent sur l'histoire et la philosophie des sciences. Cela n'est pas non plus ce que je crois. Laissez moi clarifier cela. Je crois (...)

Contrairement à des formes traditionnelles de textes écrits, ces formes d'écriture montrent le processus de construction des arguments en interaction avec les destinataires de l'argumentation. Le débat est réécrit au fur et à mesure de son évolution. Les messages sont mélangés, tissés ensemble pour aboutir à une sorte de position résultante, à vrai dire bien confuse. En fait, cette appropriation collective des messages de certains participants aboutit à une ré-appropriation de la légitimité et de l'importance des arguments défendus par d'autres participants. Ceux qui cherchent à imposer leur position dans le débat ont intérêt à maintenir cette impression d'une élaboration collective, c'est-à-dire impersonnelle. Leurs arguments sont, de ce fait, acceptés plus facilement par la communauté, tandis qu'ils gagnent en même temps en légitimité. Autrement dit, une stratégie possible est d'utiliser la dynamique de construction d'une position commune, illustrée par l'écriture quasi-orale, à travers une pratique de synthèse de messages, pour asseoir son autorité dans le débat. Cela revient à utiliser l'écriture comme s'il s'agissait d'une parole, mais en tirant parti de l'écriture et du fait qu'elle renvoie à un auteur et non pas à un collectif.

Le lien entre l'écriture et l'oralité apparaît ici clairement : les techniques de l'écriture utilisées ici pour prendre le pouvoir dans le débat sont les techniques habituellement propres à l'oral : ce sont celles qui consistent à reprendre en résumant les arguments de ses adversaires tout en les reformulant à sa manière et à son avantage, ou encore à utiliser l'effet de contexte créé par un ensemble de messages se répondant sur une question 
pour être capable de formuler une synthèse apparemment "à chaud". De plus, l'interaction électronique avantage encore plus l'auteur/orateur par rapport au lecteur/public. Tandis que dans l'échange verbatim, il est généralement possible de reprendre les paroles d'autrui pour préciser les points qu'il aurait déformés, dans l'échange écrit, même "interactif", il est beaucoup plus difficile, comme nous l'avons vu, de reprendre le texte d'autrui. Mettre en avant la quasi-oralité de l'écriture électronique est donc tout à l'avantage de ceux qui tentent de prendre le pouvoir sur les échanges. Ces derniers utilisent pour imposer leur position l'effet de communauté créé par la quasi-oralité, effet espéré par les participants, en même temps que les techniques de l'oral, effet masqué par l'attente d'oralité des membres de la liste.

\section{Actions tactiques et actions stratégiques}

On peut donc distinguer ceux qui, d'une part, ont réussi à devenir des "leaders" dans le débat à travers un travail de reformulation et de synthèse, et ceux qui, d'autre part, ont tenté d'utiliser les effets de contexte du débat pour formuler leur position au moment qui apparaissait opportun. Je présenterai ces deux positions de prise de pouvoir en termes de stratégie et de tactique. Certains détails de l'analyse de contenu du débat indiquent clairement cette opposition, comme l'échange entre Steve Fuller et Sharon Traweek (que l'on peut respectivement qualifier de stratège et de tacticienne ${ }^{39}$. Traweek était la seule femme du groupe de participants actifs qui soit intervenue fréquemment. Le message suivant est la réponse de Traweek à un message que Fuller lui avait adressé et dans lequel il commentait un autre de ses messages. Il ne renvoie pas uniquement à un désaccord sur des questions académiques, mais à des différences dans la manière de défendre sa position.

\footnotetext{
39. Nous pouvons replacer cet échange dans le contexte académique plus large de la communauté STS. Dans l'ouvrage Science as Culture and Practice édité par Andrew Pickering (PICKERING, 1992), les contributions sont destinées à produire une synthèse des différentes approches et des perspectives qui composent le champ STS. Le volume comprend une contribution de Fuller (FULLER, 1992) et une autre de Traweek (TRAWEEK, 1992). Leurs positions s'éloignent l'une de l'autre autant qu'il est possible, comme l'indique Pickering dans un chapitre introductif à ces contributions. Le débat électronique a parfaitement illustré cette tension et l'a rendue plus manifeste et bien plus vivante que dans un article traditionnel.
} 
Date: Wed, 26 Oct. 1994 ; From: Sharon Traweek ; Subject: discussion entre différentes sortes de praticiens de la recherche

“ (...) Je suis extrêmement réticente à répondre aux remarques de Fuller, non seulement parce que cela nécessiterait un long message, mais également parce que, à moins d'être prudente, cela le mettrait de fait dans la position de définir les termes de la discussion, ce qui, bien entendu, est le problème (...)

En ce sens, je vais simplement intercaler mes remarques dans ce que Fuller a dit à propos de mon dernier message. Dans un ultime message, bien entendu,

Le Merc. 26 oct. 1994, Steve Fuller a écrit : [il s'agit d'un insert automatique du logiciel de lecture de courrier électronique : ]

$>$ En ce qui concerne TRAWEEK, je ne voit pas clairement quel modèle

$>$ d'interaction STS-scientifiques elle utilise.

(...) Rhétoriquement, l'expression de Fuller "(au moins mon) manque de clarté" est une démonstration plutôt claire reconnaissable à quiconque est familiarisé avec certaines manifestations du débat antagoniste académique. Pour les non initiés, je l'explique : l'implication est que 'TRAWEEK' manque de clarté. Le 'à première vue' dit explicitement que je ne suis pas parvenue, dans mes messages, à rendre clair même le premier point concernant la raison pour laquelle 'les propos de TRAWEEK, oralement ou par écrit, seraient intéressants pour les scientifiques'. Nous sommes là au stade du stylo rouge dans la marge; c'est-à-dire, j'ai été classée comme la mauvaise élève. En éthologie, ce type de comportement s'appelle un mouvement de domination. J'abrège.

(...) Revenons à Fuller [oups, cela devrait être FULLER] qui d o u c e m e n t a posé un cadre de questions à “ TRAWEEK " comme si j'étais encore l'étudiante sans finesse qui reçoit sa copie biffée de rouge (pourquoi les lecteurs de cette liste acceptent-ils cela alors que leurs messages sont disséqués d'une manière aussi sèche ?) :

(...) Ah, oui, le logo de conclusion de FULLER :

$>$ A vous, en discours,

$>$ Steve Fuller

Je me suis toujours demandée : discours avec qui ? De quel genre de discours s'agitil ? Que suis-je arrivée à faire de mon message ce soir ? Est-ce que ce palimpseste appelle un commentaire ? Les commentaires de FULLER et mes intercalements forment-ils un duo ? De quelle genre d'interaction s'agit-il ici ? Ce qui nous ramène ici au début..."

L'action de Fuller, que je qualifierai de stratégique, se fait à travers la mise en place d'une vision particulière sur la situation - en l'occurrence sur des questions telles que: quel est le rôle du mouvement STS ? Qui peut légitimement parler au nom de ce mouvement? Pour construire une telle 
position à partir de laquelle exercer un pouvoir, il est nécessaire de trouver une rhétorique pouvant satisfaire, au moins en surface, tous les participants. Dans le cas décrit ici, Fuller voulait présenter la "vraie " représentation du mouvement STS, pour ainsi pouvoir s'en servir en fonction de ses propres arguments. A l'inverse, l'attitude de Traweek, que l'on peut qualifier de tactique, a consisté à trouver des occasions pour donner au cours des échanges des possibilités d'expression à un plus grand nombre de personnes. Fuller a utilisé un discours académique et rhétorique, faisant essentiellement appel à la culture écrite, ceci pour renforcer sa position à l'encontre de Traweek. Cette dernière a utilisé au contraire le texte même de Fuller - dans l'idée d'une interaction orale - pour élucider la manière stratégique dont il était construit. Traweek a déconstruit à la fois l'attaque de Fuller et sa stratégie discursive.

Le dispositif de communication est en somme utilisé de deux façons antagonistes, correspondant à deux politiques de pouvoir - stratégie et tactique - et à deux conceptions de l'écriture - quasi-orale ou une écriture en mosaïque. En effet, les pratiques de synthèses de messages et de (re)définition du contexte d'énonciation d'autres messages renvoient à une activité purement scripturale, tandis que les actions tactiques d'utilisation du contexte et de l'évolution du débat tirent davantage parti des interactions qui ont lieu. Ainsi, d'une part, le dispositif donne le sentiment d'une potentialité pour élaborer un sens de la communauté, et, d'autre part, il fait exister de nouvelles possibilités pour exprimer des revendications individuelles sur la "bonne " manière d'interpréter les formulations multiples qui résultent de la discussion. Cette tension peut être considérée comme une illustration de la confrontation entre les répertoires de l'oral et de l'écrit.

L'approche qualifiée ici de tacticienne a consisté à utiliser les occasions fournies dans le déroulement des échanges pour expliciter le contexte de ces échanges et pour tenter de rendre les autres membres conscients des enjeux de la discussion. Parfois, une participation plus large des membres de la liste était rendue possible du fait de ces initiatives. L'idée sous-jacente à une telle procédure était de constituer une forme de contre-pouvoir face aux volontés de contrôle de certains participants. A l'opposé, l'action stratégique a consisté à utiliser l'illusion d'une interaction quasi-orale pour pouvoir manipuler d'autant mieux l'écriture et les effets d'ironie, dus à l'absence d'un référent commun, qui en ont résulté. Là où l'action tacticienne tentait de faire vivre la quasi-oralité, même imaginaire, l'action stratégique a essayé de l'utiliser à travers l'écriture académique et rhétoricienne. En ce sens, 
utiliser l'écriture quasi-orale - si elle correspond à une tentative de créer du lien social pour une communauté - peut également être un moyen pour ne pas dire le leurre de la communication électronique (celui de la sincérité, de la spontanéité, de la transmission fidèle d'un sens, etc.). Considérer l'échange électronique comme une conversation peut être tentant, mais laisse dans l'ombre la machinerie de l'écriture qui consiste justement à construire ce style conversationnel.

Ainsi, il ne faut pas oublier que la communication orale, ou quasi-orale, n'est pas plus transparente que la communication écrite. Goody 40 l'indique clairement: " Ce n'est que par une abstraction commode, mais génératrice d'illusions, qu'on peut traiter la parole comme un simple échange réciproque entre partenaires équivalents. Tout sens est le produit d'un jeu social de construction de sens, d'un déchiffrement à plusieurs niveaux, où s'affrontent des positions d'inégal pouvoir". En somme, qu'il s'agisse d'une production écrite, orale, ou quasi-orale, l'exercice de l'autorité demeure un élément difficile à contourner. Or ici, cette autorité n'apparaît pas nécessairement - sauf à être dénoncée comme le fait précisément Traweek - du fait d'une mise en scène orale et tactique des échanges. L'élaboration collective a été accentuée, tactiquement ou stratégiquement en fait, laissant dans l'ombre les stratégies individuelles.

Cette opposition peut encore être rapprochée de ce que Sherry Turkle appelle, en reprenant les études culturelles américaines : les " cultures" des hommes et des femmes. Nous pouvons comparer les attitudes stratégiques et les attitudes tactiques en fonction des réactions des hommes et femmes face à l'utilisation de l'informatique. Bien que la comparaison soit caricaturale, elle est néanmoins suggestive. Turkle a observé l'existence de deux approches fondamentales de l'usage de l'ordinateur. Elle a identifié ce qu'elle appelle d'une part les " maîtres durs" (hard masters) et d'autre part les "maîtres doux" (soft masters). Les premiers appliquent un style structuré et technique pour imposer leur volonté sur la machine, tandis que les seconds adoptent une attitude plus interactive pour permettre aux idées d'émerger graduellement. L'auteur a constaté que la plupart des " maîtres durs " étaient des garçons alors que les " maîtres doux " étaient plutôt des filles. Dans le cas qui nous intéresse ici, on peut retrouver une part de cette distribution sociale des rôles, puisqu'une seule femme a pris une place

${ }^{40}$. GOODY, 1979, p. 26.

${ }^{41}$. TURKLE, 1986. 
importante dans le débat. De plus, plusieurs autres femmes ont choisi de ne pas participer au débat, comme elles l'ont expliqué en réponse à mon questionnaire. Cette attitude ne signifie pas, bien entendu, qu'elles n'avaient rien à y apporter, mais on peut dire que ce mode d'action peut être rapproché d'une attitude tactique d'analyse des échanges, c'est-à-dire d'une participation moins visible sur la scène du débat en ce sens qu'elle utilise les opportunités de "prise de parole" qui se présentent ou non au cours des échanges.

Il convient donc de rester prudent: ce n'est pas parce qu'existe en permanence un espace où peuvent se formuler des éléments d'un débat scientifique sans que pèse la contrainte d'une formalisation rigoureuse, qu'il va nécessairement en ressortir des effets positifs pour la pratique, un renforcement des liens dans la communauté, une avancée dans la compréhension des problèmes et dans la synthèse entre positions différentes, etc. Ce n'est pas le nombre de positions différentes qui fait défaut dans ce type de débat, mais bien le manque de discernement qu'il est possible d'exercer à travers ce mélange de préjugés, de positions partisanes, de revendications diverses et d'intentions véritables d'éclaircir certaines questions, sans qu'il soit véritablement possible de trouver le registre dans lequel les messages sont à interpréter. Puisqu'il n'y a plus un texte censé porter une idée et servant de référence, mais plutôt des collages de textes, le lecteur peut être tenté de chercher les fragments qui le confortent dans sa position, au lieu d'avoir à faire l'effort de compréhension et éventuellement de "traduction" de la pensée d'autrui ${ }^{22}$. Cette situation favorise une surenchère de commentaires, sans pour autant produire un enrichissement des positions de chacun. Ce qui disparaît ici, paradoxalement, c'est une capacité de mise en perspective des récits, des énoncés, alors que ceux-ci se bousculent dans les forums de discussions. En un sens, nous retrouvons le problème de l'hypertexte : l'hétérogénéité des lectures possibles est mise en scène et balisée par un dispositif technique, mais les pratiques interprétatives des lecteurs ne s'en trouvent pas forcément renforcées.

\footnotetext{
42. Il ne s'agit pas ici d'une pratique telle que peut avoir, par exemple, un spécialiste d'un domaine qui recherche une information factuelle précise, mais concerne un autre niveau de communication : les messages portent sur des discussions qui font appel au sens commun et non des faits spécifiques concernant des résultats scientifiques ou techniques. Rob Kling et Charles Dunlop dénoncent cette dérive qu'ils constatent dans certains forums de discussions publics. KLING, DUNLOP, 1991.
} 
Les participants à un débat électronique peuvent donc légitimement douter de la véracité des énoncés qui se succèdent sans se suivre, et se trouvent, d'une manière générale, face à une masse discursive qui ne leur apporte parfois qu'une éventuelle confirmation de leurs propres prises de position, sans pouvoir les "jauger" face à la communauté (réelle, et non pas électronique).

Conclusion? L'écriture quasi-orale correspond certes à une tentative pour remédier à l'absence de référent, mais s'engage par là dans des jeux de pouvoir sur la bonne interprétation de la situation débattue. Au lieu d'éclaircir la situation, le débat électronique semble au contraire la complexifier. Mais qu'en est-il dans le cas où ce type de dispositif est utilisé par une communauté locale ? L'absence de référent est-elle effectivement compensée par la "présence" effective d'une communauté de recherche ? Dans le cas où l'espace de discussion électronique est investi par un collectif en liaison avec un vécu social commun et quotidien, que peut-on dire des conséquences qui en découlent pour le collectif, pour l'élaboration d'un espace de dialogue ? Afin de tenter de répondre à ces questions, je rendrai compte d'un second débat électronique, au sein d'une faculté de chimie, dont les membres ont considéré l'aboutissement comme étant positif (mais à quel titre et sur quel plan ?). Une observation rapide de ce forum nous permettra de formuler quelques hypothèses sur ce succès.

\section{LA LISTE “CHIMIE ” COMME ESPACE DE REFORMULATION DES DIFFÉRENCES}

L'élément nouveau et essentiel introduit par le cas de figure de la liste des chimistes est la présence d'un référent externe aux textes échangés à travers Internet, qui vient donner sens à l'implicite présent dans les messages et qui limite par là même les possibilités de manipulations stratégiques. Nous verrons ici, sans entrer dans les détails de l'analyse du débat, comme nous l'avons fait dans le cas du débat STS, comment la présence d'un contexte local peut permettre de remédier aux limites du dispositif, sur le plan de la compréhension implicite du sens des échanges. Mais peut-on pour autant situer l'apport d'un tel espace de discussion sur le plan du développement du sens du collectif, ou encore de l'acquisition de savoir nouveau ? Comme nous allons le voir, les membres de la liste n'ont pas formulé ces apports dans ces termes. Cette dernière partie se présentera donc comme une série d'hypothèses sur l'usage de ces forums électroniques dans un contexte local. 


\section{Présentation de la liste chimie}

La liste électronique concernant la chimie regroupe l'ensemble des chimistes strasbourgeois. Elle représente en quelque sorte un forum pour l'ensemble de la communauté, et constitue le moyen le plus efficace pour diffuser très rapidement un message à tous les chimistes. Malgré la perte de temps occasionnée par la lecture de messages parfois non pertinents, la liste " chimie" a été globalement perçue comme étant très utile : ce qui est un signe qui ne trompe pas, lorsque l'on connait la grande distance qui existe parfois entre la pratique de la chimie et l'usage d'un ordinateur. En particulier, elle a été considérée comme pouvant servir de relais auprès d'une communauté et constituer des " ponts" entre laboratoires. Il y a bien sûr un intérêt très pratique à ce type de liste. Par exemple, certains utilisateurs de la liste envoient des messages du type: "cherche 1 gramme de ceci ou cela" en considérant que pour tous les "chimistes de paillasse" ces messages sont importants, en gain de temps et économiquement. Mais ce qui est étonnant, c'est que cette liste de discussionS ne s'est pas limitée à ce type d'échanges. En effet, il s'y est déroulé un débat de fond, portant sur des questions de nature épistémologique et concernant l'ensemble de la communauté des chimistes. Cette discussion a démarré sur une question de distinction dans le domaine de la chimie physique entre praticiens classés en physico-chimistes et en chimico-physiciens, question soulevée initialement en liaison avec la réorganisation des enseignements en DEUG au sein de l'université. Elle a servi de point de départ à un questionnement plus large portant sur - Jes diverses manières d'aborder sa pratique au sein d'une même discipline ${ }^{43}$. Ce type d'échanges et de débat est nouveau, non pas

\footnotetext{
${ }^{43}$. Voici quelques données permettant de mieux situer le débat analysé ici : la faible quantité de messages (38 messages envoyés entre le 12 février 1996 et le 13 mars 1996), contrairement au débat STS (230 sur 10 semaines), ne permettait pas de faire une analyse exhaustive des stratégies d'intervention dans le débat. C'est davantage l'interaction du débat électronique avec la vie de la communauté qui est intéressante à étudier car elle permet de comprendre les manières de créer et d'investir sur le plan local un espace de dialogue nouveau. La discussion a débuté le 8 février 1996: après une première rencontre au sein de l'UFR de chimie, un message sur la liste "chimie" annonce une réunion de réflexion sur la restructuration des licences et maîtrises de chimie et chimie physique prévue le 15 février. Le 12 février, un premier message - assez détaillé - lance le débat sur la définition de la chimie physique. Son auteur propose également en conséquence une organisation des modules d'enseignement de cette discipline. Une série d'échanges a lieu en réponse à ce message. Il est à noter que dès le début, les messages font référence en permanence à des réunions se déroulant en parallèle, préparant ainsi la réunion du 15 . Le compte rendu de la réunion du 15 est envoyé à la liste le 22 février. Celui-ci relance la discussion et propose deux autres dates de rencontres pour
} 
simplement à cause du média employé, mais surtout parce qu'il ne s'était tout simplement jamais produit auparavant, même lors des différentes rencontres qui rythment la vie d'une communauté de recherche au sein d'une université. De plus, la liste n'était pas destinée, à l'origine, à être le support d'échanges autour des pratiques de chacun, ni de réflexion sur les différences d'approches au sein de la discipline scientifique. Il est donc très intéressant pour notre analyse de rendre compte des raisons d'un tel développement.

La nouveauté de ce débat témoigne d'une réappropriation de la ressource en fonction de la dynamique sociale d'une communauté d'utilisateurs. On ne peut pas séparer arbitrairement le fond de la forme : si ce débat a émergé sur la liste "chimie", et non pas dans des réunions rassemblant différents membres de la communauté des chimistes strasbourgeois, le média a probablement joué un rôle déclencheur. Reste à comprendre ce rôle.

\section{Un espace de dialogue local}

Une analyse du calendrier des interventions dans le débat électronique des chimistes montre qu'elles alternent avec les différentes rencontres (en présentiel) entre chimistes de la communauté strasbourgeoise. La liste de discussions de chimie a servi de relais à des rencontres qui se sont déroulées en parallèle au sujet de l'enseignement de la chimie physique à l'université. Le débat électronique préparait, développait et résumait des questions posées dans les réunions entre chimistes. Inversement, le débat électronique a été mentionné durant les réunions comme étant une extension, ainsi qu'une manière de placer sur un plan différent, de ce qui était en jeu dans cette réforme. Si les échanges électroniques se font dans un état d'esprit différent, ils sont néanmoins validés :

“Je me demande si, une fois que vous aurez réuni toutes ces opinions, leur total (plus éventuellement une conclusion) ne donnerait pas matière à un intéressant article pour l'Actualité Chimique?"

poursuivre le débat: le 23 février et le 4 mars. Le débat est repris le 22 février, pour se terminer le 13 mars. Si les chiffres de la participation effective au débat sont comparables à ceux du débat STS (20\%), les membres de la liste "chimie" étaient tous les chimistes de l'université. Ceux-ci étaient, en effet, automatiquement inscrits dans cette liste à partir du moment où ils avaient une adresse électronique (ce qui était le cas pour l'ensemble des chercheurs). 
On pourrait ainsi rapprocher ce débat d'une utilisation du dispositif en travail collaboratif (le Computer-Supported Collaborative Work). Cependant, la faible quantité de messages et l'absence de projet commun nous obligent à préciser la fonction d'un tel espace d'échange. Il ne s'agit pas véritablement d'un outil de travail collaboratif, ni d'un espace de débat autour d'enjeux globaux, stratégiques et politiques, comme dans le cas du débat STS. Ce qui caractérise le débat des chimistes se situe davantage sur le plan de la possibilité de reformuler "publiquement" les positions de chacun et "d'expérimenter" les différences qui existent dans une communauté de recherche locale.

Le caractère public de ces déclarations électroniques est problématique, nous l'avons vu pour le débat STS. Mais c'est peut-être justement là que réside l'avantage de ce type d'interactions dans une communauté locale de recherche. En effet, si ce dispositif est utilisé dans un collectif qui a tout loisir de se rencontrer et de débattre de tous les problèmes pouvant se poser à lui, on peut légitimement penser que l'avantage retiré porte sur le rapport particulier au public induit par les forums électroniques. Nous avons évoqué le problème que posaient les lurkers dans ce type d'interactions. Mais ces derniers sont néanmoins davantage "présents" dans ce type d'échange, car chacun sait que tous les autres recevront tous les messages, mais ne les liront pas forcément. Ainsi, se combinent ici des éléments de l'échange institutionnel d'une communauté de recherche avec les échanges informels, les discussions de couloirs ou de cafétéria. Il s'agit d'un moyen officieux de faire savoir à tous sa position, sans avoir pour autant à l'assumer directement, comme dans une assemblée, ni avoir à rendre compte ultérieurement des traces écrites de cette prise de position. Les "inconvénients" de l'oral public et de l'écrit diffusé se retrouvent ainsi gommés à travers ce mode d'échange hybride.

Cependant, cette dilution du risque - celui de la prise de parole - peut en fait aboutir à une utilisation du dispositif, non pas en vue de créer des collaborations et de développer une communication organisée autour de projets, mais pour en faire une sorte d'espace permissif, dans le sens où il est possible de dire tout haut, non sans ironie et provocation, ce que l'on se contente de penser pour soi dans les contextes sociaux habituels :

" Soyons logiques, si chimico-physique est un terme horrible alors physicochimie est nécessairement un terme atroce (et quid de la bio-physico-chimie). Quand au fonctionnement des neurones, il faudrait être plus précis : quelle 
est l'activité la plus sollicitante pour les neurones, la chimie ou la chimiephysique?"

Un tel espace peut être intéressant dans un contexte professionnel et pour une communauté de recherche: il devient possible de suivre, reprendre et réinterpréter les activités qui rythment une communauté de recherche, dans une logique de travail coopératif, et, en même temps, de ne pas vraiment s'impliquer, de tester certaines positions sans prendre réellement parti, de dire également son spleen à tout moment sans avoir à trouver un collègue bienveillant, de suivre les échanges, disputes ou bruits de couloir sans être pris à partie, etc. En somme, le dispositif permet de suivre la vie d'une communauté, et d'avoir le sentiment d'en faire partie, de contribuer à son quotidien, sans avoir à le vivre jusqu'au bout : en ce sens, chaque membre de la liste se trouve dansfa position de suivre un feuilleton, tel une série TV, mais avec ses collègues ${ }^{4}$...

Nous retrouvons ici en quelque sorte les conclusions de Kiesler et Sproull, à savoir que le manque d'indices sociaux dans les échanges électroniques permet une participation plus large que dans des interactions menées en face à face, mais en y apportant un certain nombre de précisions : ce phénomène repose, selon mon hypothèse, davantage sur le type d'écriture qui s'y développe (contextuelle, sans réelle pérennité, comme la parole) que sur le plus grand anonymat de l'échange. De plus, c'est le contexte d'usage qui détermine largement les effets du dispositif qui seront utilisés : le vague et l'inconséquence de l'écrit électronique dans le cas présent, les effets de quasi-oralité dans le cas du débat stratégique précédent. On peut probablement repérer d'autres régimes d'usage du dispositif de communication électronique: des logiques d'entraide, notamment entre scientifiques du brain-gain et scientifiques restés dans les pays en voie de développement, des logiques de maintien de l'ambiguïté dans l'échange, en particulier lorsqu'on adresse une demande à un supérieur hiérarchique, des logiques de séduction, la drague sur Internet, etc. On voit que ce n'est pas simplement l'absence d'indices socioculturels qui permet de déployer ces tactiques ou stratégies. Ces indices réapparaissent d'ailleurs partiellement

\footnotetext{
${ }^{44}$. Ce type de pratiques apparaît de façon beaucoup plus radicale à travers les webcams, ces caméras branchées par des amateurs sur leur site Internet, les montrant dans leur environnement quotidien, offrant parfois aux visiteurs virtuels leur intimité, dans une logique voyeur/exhibitionniste. Il me semble que le suivi des débats électroniques peut mettre les membres non participants (les lurkers) dans une position similaire à celle des webcams.
} 
dans la langue utilisée et les formulations, mais c'est bien l'indétermination, peut-être la liberté au niveau de l'écriture qui prime.

Mais au-delà de cette fonction des échanges électroniques que l'on pourrait rapprocher de la parole phatique, quelle fonction ces échanges peuvent-ils remplir sur le plan de la pratique scientifique ? Les messages relevés au cours du débat indiquent qu'ils n'étaient pas sans intérêt sur le plan du contenu :

“ La chimie-physique est un état d'esprit, tout comme la chimie, mais quelque peu différent (les neurones ne fonctionnent pas exactement de la même manière), mais les deux sont heureusement complémentaires."

"Le découpage ne doit pas exister. [...] Dans la pratique de tous les jours, il est clair qu'il y a continuité et qu'il vaut mieux ne pas insister sur ce qui différencie mais plutôt sur la complémentarité de chacun."

"Le clivage chimie-organique chimie-inorganique est un peu dépassé."

“ La chimie du solide souffre de sa préhistoire et c'est aux chimistes du solide de démontrer aux molécularistes qu'ils n'ont pas le monopole de la créativité en chimie. " [...] " Dans toute tentative de classification, il y aura des contradictions inévitables. N'est-ce pas là une manifestation éclatante du théorème d'incomplétude de Gödel dans le domaine de la chimie ? [...]."

Ainsi, si cet espace d'échange un peu flou a été investi par les chimistes, on peut formuler l'hypothèse qu'il a pu jouer un rôle pour leur pratique quotidienne. Cette interprétation s'appuie sur les possibilités d'expression offertes par la liste étant donné le cadre particulier des échanges. En effet, contrairement au cas de la liste STS, il n'y a pas de confusion entre le texte vraisemblable, la description d'une situation et la prise de position individuelle. De plus, il y a ici des référents externes, implicites et socialement incorporés dans les pratiques et la langue, auxquels renvoie leur écriture. Certes, le forum a pu constituer une sorte de défouloir, après les réunions de travail, pour exprimer tout ce qu'il n'avait pas été possible de dire. Mais il a également fonctionné pour les chimistes comme un espace de reformulation d'éléments de leur pratique quotidienne. En outre, il est peutêtre important que ces deux dimensions, apparemment contradictoires, aient pu être développées à travers le même dispositif. De fait, il devient ainsi possible de dire sa pratique, hors de toute contrainte formelle, par exemple celle de l'écriture d'articles ou de présentation à des colloques, même si ce dire n'est qu'une tentative maladroite et parfaitement inutilisable pour les collègues. En effet, c'est cette nécessité de donner du sens à ce qui s'élabore 
localement sur une paillasse ou autour d'un appareillage qui garantit que les scientifiques ne sont pas victimes d'hallucinations. Un énoncé formel seul ne peut garantir la validité de leurs observations.

Au surplus, les participants n'étaient pas condamnés à simplement formuler leurs positions: ces confrontations entre textes, loin de mettre en pièces toute interprétation possible, pouvaient constituer au contraire autant d'invitations à aller voir son collègue du laboratoire voisin, pour lui demander plus précisément de quoi il parlait ! C'est d'ailleurs ce que certains participants au débat ont explicitement suggéré : ainsi, une personne a notamment voulu répondre publiquement à une critique qui lui avait été faite par un collègue (sérieusement ou non ?), lui reprochant de réagir " comme un physicien ". Lui même se définissant comme étant pleinement un chimiste, sa réponse sur la liste fut la suivante :

"J'invite tous ceux qui auraient des doutes sur la nature de mon activité ici à Strasbourg, à venir visiter mon laboratoire. Ils n'y trouveront que des étudiants manipulant des solutions dans le but d'obtenir de nouvelles molécules précurseurs de nouveaux matériaux. Si vous décidez qu'il s'agit d'une activité typique de physicien, alors moi je n'ai plus qu'à me faire curé. Si par contre vous décidez qu'il s'agit bien là d'une activité de type chimique, alors je me pose la question des motivations qui poussent certains chimistes à traiter d'autres de physiciens."

Un travail de reformulation de leur activité est indispensable aux scientifiques pour que leurs énoncés puissent être repris ultérieurement dans leur pratique. Celle-ci s'effectue lorsque les chercheurs discutent avec leurs collègues autour de leur travail. Lorsqu'ils interprètent ce qui s'établit au travers de leur pratique, ils reprennent "concrètement" à la fois les énoncés formels et ajustent une pratique avec une position d'un collectif de pensée.

On peut rapprocher cette activité, que je qualifierais volontiers de pratique d'acculturation, à ce que Pickering appelle mangle 45 . Ce terme décrit la manière dont la science se définit comme une zone de rencontre entre machines, façons de faire, théories, faits, relations sociales qui interagissent dans ce qu'il appelle "the mangle of practice". Il étend ainsi la notion de culture scientifique aux savoir-faire et aux relations sociales, et non pas uniquement à un champ de savoir. Cette notion permet de montrer

45. PICKERING, 1995. 
l'ajustement fait par les scientifiques entre la réalité vécue au laboratoire et les positions formelles à défendre au niveau d'un collectif de pensée, ou entre collectifs, au sens de Fleck $\square$

Il est donc possible de dire, au vu du débat entre chimistes, que c'est justement cette seconde catégorie de production scientifique qui trouve à travers Internet de nouvelles possibilités d'expression. A condition qu'un référent puisse effectivement être désigné, donc à condition qu'un collectif réel, partageant un sens commun, utilise l'espace de discussion, le travail d'hybridation, de formulation et de reformulation qui s'expérimente au quotidien de la pratique scientifique, peut trouver à travers Internet de nouvelles ressources pour enrichir la pratique hétérogène qui caractérise dans les sciences l'activité d'acculturation.

Ce travail de reformulation peut être rapproché de l'activité de vulgarisation scientifique, dans le sens où celle-ci participe du travail d'acculturation des énoncés scientifiques hors du collectif et dans la mesure où elle permet une reformulation des énoncés scientifiques en ayant recours à une connaissance du monde familière et commune ${ }^{477}$. Cependant, les textes de vulgarisation constituent en général un discours qui n'est pas référé à la pratique effective des chercheurs, ils relèvent d'une reconstruction a posteriori qui ne correspond pas à la dynamique dans laquelle s'engagent les scientifiques au nom des questions très précises qu'ils posent. Ainsi, l'activité de reformulation sur Internet, articulée à un collectif, permettrait-elle, mais ce n'est là qu'une simple hypothèse, de produire ce travail de reformulation sans passer par la forme conventionnelle de la vulgarisation scientifique.

Ce ne sont donc pas les effets de quasi-oralité, tels qu'ils ont été identifiés dans le débat STS, qui ont servi à développer dans le cas présent un sentiment de communauté et de partage d'un sens commun, mais bien l'activité de reformulation de la pratique de certains au sein du collectif. Ainsi, l'impression de faire partie d'une communauté à travers Internet, si elle ne s'accompagne pas d'une existence de relations sociales réelles, constitue une illusion due au support même de l'information qui lui donne sa validité. En revanche, inscrit dans une communauté de recherche, on peut dire que le réseau devient un moyen de mener un travail de construction de sens à partir des pratiques de chacun au sein du collectif.

${ }^{46}$. FLECK, 1979, op. cit.

${ }^{47}$. Voir JURDANT, 1993, pp. 365-373. 
L'apport de ces échanges se trouve, pour les scientifiques, dans la possibilité de formuler publiquement leur pratique dans des termes non spécialisés. Sur le plan de la communauté, il ne s'agit donc pas là de développer par ce dispositif une participation effective à un collectif, mais, sans l'implication que suppose sa présence physique dans un espace collectif, de pouvoir assister à la vie d'une communauté et d'avoir la possibilité de reformuler ce qui fait sens à la fois pour soi et pour un collectif de pensée particulier, au sein d'un espace qui convoque une communauté élargie à plusieurs collectifs, cela sans avoir à engager le débat scientifique sur le sens précis de chaque mot employé.

\section{CONCLUSION}

Il n'est jamais clair et il est même parfois impossible d'établir les enjeux implicites lorsque des personnes participent à des échanges. Nous pouvons seulement faire des suppositions fondées sur les interactions observées. Mais lorsqu'un tel débat se déroule sur plusieurs mois et lorsqu'il se produit au travers d'une technologie de l'écrit particulière, pouvons-nous encore nous fonder sur la compréhension implicite que nous avons d'un débat, quel qu'il soit ? La forme du débat électronique induit certes une continuité dans le style avec d'autres formes d'écritures (note de synthèse, note de lecture, texte d'une conférence, etc.). Cependant, ce mode d'écriture fait exister des formes d'interaction qui se rapprochent des propriétés de l'échange oral. A travers les pratiques de reprises, de citations, de synthèses, à travers l'utilisation du contexte des échanges, à travers également le déroulement temporel du débat en parallèle avec d'autres événements, se construit cette quasi-oralité de l'écriture qui fait apparaître le vraisemblable dans le texte.

Le recours aux notions de quasi-oralité et de vraisemblance, dans l'analyse de cette forme d'interaction permet de situer les enjeux et limites de ces pratiques de communication. Une approche qui se limiterait aux rapports de forces et à la construction d'un sens de la communauté au sein des forums électroniques manquerait un point essentiel, le fait que le défaut de référent commun dans le texte produise pour y remédier confusions et tentatives tacticiennes. La quasi-oralité est l'une de ces tactiques. Elle consiste dans ce travail sur l'écriture visant à y introduire des propriétés de l'échange oral, comme la participation collective à ce qui s'énonce, la définition en situation du contexte de l'échange, un sentiment de communauté, autant de 
manières de ne pas faire éclater aux yeux de tous l'absence de référent commun et l'incohérence du discours.

Loin de faire exister un sentiment de communauté, l'écriture quasi-orale participe de politiques de pouvoir qui agissent sur la construction du débat. Nous sommes ici bien loin de certains propos sur la "civilité assistée par ordinateur" de Pierre Lévy selon laquelle cet espace de dialogue créerait une "nouvelle démocratie [qui] pourrait prendre la forme d'un grand jeu collectif dans lequel gagneraient (mais toujours à titre provisoire) les plus coopératifs, les plus urbains, les meilleurs producteurs de variété consonante... et non plus les plus habiles à prendre le pouvoir, à étouffer la voix des-autres ou à capter des masses anonymes dans des catégories molaires ${ }^{48}$,

Le contexte d'un débat électronique se met en place à partir des citations de messages. Mais contrairement à l'oral ou toute reprise est une reformulation, les reformulations sont ici stratégiques, puisqu'elles se fondent sur l'écrit et la reproduction à l'identique qu'il permet. De même, la création d'un sentiment de communauté et d'un échange sur un mode oralisé est utilisé pour pouvoir recourir aux techniques de la persuasion orale, sans que ces dernières apparaissent comme étant suspectes ou incongrues.

En revanche, dans le cas d'une communauté locale, ou d'un collectif amené à se rencontrer très régulièrement, l'existence d'un référent commun permet d'étendre les échanges à un travail d'acculturation. Si de telles pratiques ne permettent pas de participer réellement à la vie de la communauté, puisqu'il s'agit plutôt de l'observer et de tester certains arguments, elles renvoient cependant à une phase importante de l'activité scientifique. Cela ne signifie pas pour autant que l'interaction directe ne soit plus un passage indispensable à cette acculturation. Il s'agit au contraire de la possibilité de son articulation avec un niveau plus large, autrement dit de pouvoir rapprocher à certains moments l'activité locale d'un laboratoire avec la nécessité de faire sens à un niveau plus large, intercollectif. Il ne s'agit pas pour autant d'un passage soit par la forme institutionnelle de la vie d'une faculté d'enseignement et de recherche, soit par la forme de la vulgarisation scientifique. Dans ce cas de communauté locale, Internet fait exister une forme de médiation qui permet à des chercheurs d'articuler, sous certaines

${ }^{48}$. LEVY, 1994, p. 75. 


\section{$46 \quad$ Réseaux $n^{\circ} 97$}

conditions, le niveau local de leur pratique avec le niveau collectif d'une communauté scientifique. 


\section{REFERENCES}

AYCOCK A., BUCHIGNANI N. (1995), “E-mail murders: reflexions on 'dead' letters", in Jones S. (ed.), Cybersociety: Computer-Mediated Communication and Community, London, Thousand Oaks and New Dehli, Sage, pp. 184-231.

BARTHES R. (1968), “L'effet de réel”, Communications, 11, Paris, Seuil, pp. 84-89.

BAYM N. (1995), “The Emergence of Community in Computer-Mediated Communication", in Jones S. (ed.) Cybersociety: Computer-Mediated Communication and Community. London, Thousand Oaks and New Dehli, Sage, pp. 138-164.

BIJKER W., LAW J. (eds.) (1992), Shaping technology - building society: studies in sociotechnical change, Cambridge, MIT Press.

DERRIDA J. (1980), La carte postale, de Socrate à Freud et au-delà, Aubier-Flammarion,

FLECK L. (1979), Genesis an Development of a Scientific Fact, Chicago, The University of Chicago Press.

FORNEL (de) M. (1994), “Le cadre interactionnel de l'échange visiophonique ", Réseaux, $\mathrm{n}^{\circ}$ 64, mars-avril, pp. 107-132.

FREEMAN L. (1984), “The impact of Computer Based Communication on the Social Structure of an Emerging Scientific Specialty", Social Networks, 6, pp. 201-221.

FULLER S. (1992), "Social epistemology and the research agenda of science studies " In PICKERING (1992), pp. 390-42.

GOODY J. (1979), La raison graphique, la domestication de la pensée sauvage, Paris, Editions de Minuit.

GROSS P., LEVITT N. (1994), Higher Superstition, Baltimore: Johns Hopkins University Press.

HERT P. (1997), "Social Dynamics of an On-Line Scholarly Debate", The Information Society, Washington DC : Taylor \& Francis, vol. 13, pp. 329-360.

HINE (1996), “Information Technology in Biology”, Society for the Social Studies of Science Annual Conference, 9-12 Octobre 1996, Bielefeld.

JEANNERET Y. (1998), L'affaire Sokal ou la querelle des impostures, Paris, Presses Universitaires de France.

JOUËT J. (1990), “L’informatique 'sans le savoir' ”, Culture technique, n²1. 
JURDANT B. (1998), Impostures scientifiques : les malentendus de l'affaire Sokal, Paris, La Découverte.

JURDANT B. (1993), “Popularisation of science as the autobiography of science ", in Public understanding of science, 2, London, pp. 365-373.

KIESLER S., SPROULL L. (1991), Connections: New ways of working in the networked organization, Cambridge: MIT Press.

KLING R., DUNLOP C. (1991), Computerization and Controversy: Value Conflicts and Social Choices, San Diego, Academic Press.

LATOUR B. (1989), La Science en action, Paris, La Découverte.

LEVY P. (1994), L'intelligence collective, vers une anthropologie du cyberspace, Paris, La Découverte.

LEWENSTEIN B. (1995), “Do public electronic bulletin boards help create scientific knowledge? The cold fusion case”, Science, Technology, \& Human Values, 2(20), pp. 123-149.

LYNCH M. (1985), Art and Artifact in Laboratory Science. A Study of Shop Work and Shop Talk in a Research Laboratory, London : Routledge \& Kegan Paul.

McLAUGHLIN M., OSBORNE K., SMITH C. (1995), “Standards of Conduct on Usenet", in Jones S. (ed.), Cybersociety : Computer-Mediated Communication and Community. London, Thousand Oaks and New Dehli, Sage, pp. 90-112.

ONG W. (1982), Orality and literacy. The technologizing of the word. London and New York, Routledge.

ORLIKOWSKI W., YATES J. (1992), “Genres of organizational communication: An approach to studying communication and media ", The Academy of Management Review 17(2):299-326.

ORLIKOWSKI W., YATES J. (1994), “Genre repertoire: The structuring of communicative practices in organizations", Administrative Science Quarterly 39(4):541-574.

PICKERING A. (1992), Science as practice and culture. Chicago: University of Chicago Press.

PICKERING A. (1995), The mangle of practice. Time, Agency and Science. University of Chicago Press, Chicago,.

SOKAL A., BRICMONT J. (1997), Impostures intellectuelles, Paris, O. Jacob.

TODOROV T. (1968), “Introduction”, Communications, n 11, Paris, Seuil.

TRAWEEK S. (1992), “Border crossings: narrative strategies in science studies as among physicists in Tsukuba Science City, Japan”, In: PICKERING (1992), pp. 429-465. 
TRAWEEK S. (1988), Beamtimes and Lifetimes, The World of High Energy Physicists, Cambridge, Harvard University Press,.

TURKLE S. (1986), Les enfants de l'ordinateur, Paris, Denoël.

VIDAL G. (1999), “Mygale-bêta : crise et décision sur l’Internet ”, Terminal, n 95, pp. 184-232.

WOOLGAR S. (1987), “ Reconstructing man and machine : A note on sociological critiques of cognitivism ", In Bijker Wiebe; Hughes Tomas, Pinch Trevor (eds.), The Social Construction of Technological Systems : New Directions in the Sociology and History of Technology. Cambridge: MIT Press. 